\title{
Structure of the fission yeast S. pombe telomeric Tpz1-Poz1-Rap1 complex
}

\begin{abstract}
Jing Xue ${ }^{1,2, *}$, Hongwen Chen ${ }^{1,2, *}$, Jian $\mathrm{Wu}^{3}$, Miho Takeuchi ${ }^{4}$, Haruna Inoue ${ }^{4}$, Yanmei Liu ${ }^{1,2}$, Hong Sun ${ }^{5}$, Yong Chen ${ }^{1,2,5}$, Junko Kanoh ${ }^{4}$, Ming Lei ${ }^{3,6}$

${ }^{I}$ National Center for Protein Science Shanghai, State Key Laboratory of Molecular Biology, CAS Center for Excellence in Molecular Cell Science, Shanghai Institute of Biochemistry and Cell Biology, University of Chinese Academy of Sciences, Chinese Academy of Sciences, 333 Haike Road, Shanghai 201210, China, ${ }^{2}$ Shanghai Science Research Center, Chinese Academy of Sciences, Shanghai, China; ${ }^{3}$ Shanghai Institute of Precision Medicine, Ninth People's Hospital, Shanghai Jiao Tong University School of Medicine, Shanghai 200125, China; ${ }^{4}$ Institute for Protein Research, Osaka University, Suita, Osaka 565-0871, Japan; ${ }^{5}$ School of Life Science and Technology, ShanghaiTech University, 100 Haike Road, Shanghai 201210, China; ${ }^{6}$ Key laboratory of Cell Differentiation and Apoptosis of Chinese Ministry of Education, Shanghai Jiao Tong University School of Medicine, Shanghai 200025, China
\end{abstract}

Telomeric shelterin complex caps chromosome ends and plays a crucial role in telomere maintenance and protection. In the fission yeast Schizosaccharomyces pombe, shelterin is composed of telomeric single- and double-stranded DNA-binding protein subcomplexes Pot1-Tpz1 and Taz1-Rap1, which are bridged by their interacting protein Poz1. However, the structure of Poz1 and how Poz1 functions as an interaction hub in the shelterin complex remain unclear. Here we report the crystal structure of Poz1 in complex with Poz1-binding motifs of Tpz1 and Rap1. The crystal structure shows that Poz1 employs two different binding surfaces to interact with Tpz1 and Rap1. Unexpectedly, the structure also reveals that Poz1 adopts a dimeric conformation. Mutational analyses suggest that proper interactions between Tpz1, Poz1, and Rap1 in the shelterin core complex are required for telomere length homeostasis and heterochromatin structure maintenance at telomeres. Structural resemblance between Poz1 and the TRFH domains of other shelterin proteins in fission yeast and humans suggests a model for the evolution of shelterin proteins.

Keywords: telomere; shelterin; hub; TRFH; evolution

Cell Research (2017) 27:1503-1520. doi:10.1038/cr.2017.145; published online 21 November 2017

\section{Introduction}

Telomeres, the natural ends of linear eukaryotic chromosomes, are essential for cell viability and genome integrity $[1,2]$. Telomeric DNA consists of short G-rich repetitive sequences and terminates in $3^{\prime}$ single-stranded G-overhangs [3]. In most eukaryotes, telomeres are extended by telomerase, a specialized reverse transcriptase that utilizes its RNA component as a template to fully

\footnotetext{
*These two authors contributed equally to this work.

Correspondence: Ming Lei ${ }^{\mathrm{a}}$, Junko Kanoh ${ }^{\mathrm{b}}$, Yong Chen ${ }^{\mathrm{c}}$

${ }^{a}$ E-mail: leim@shsmu.edu.cn

${ }^{\mathrm{b} E}$ E-mail: jkanoh@protein.osaka-u.ac.jp

${ }^{c} E$-mail: yongchen@sibcb.ac.cn

Received 12 July 2017; revised 29 September 2017; accepted 19 October 2017; published online 21 November 2017
}

replicate the ends of linear chromosomes, providing a solution to the end-replication problem [1]. Telomeres prevent chromosome ends from activating DNA damage responses $[4,5]$. Dysregulation of telomere end protection leads to the initiation of DNA damage checkpoint cascades and DNA repair activities that promote the genomic instability associated with human diseases $[3,6,7]$.

In mammalian cells, telomeres are capped by a specialized six-protein complex, shelterin, that regulates telomere length homeostasis and protects chromosome ends from degradation and end-to-end fusion $[3,8]$. In shelterin, TRF1 and TRF2 directly bind the duplex region of telomeres, and RAP1 is associated to telomeres by interacting with TRF2 [8-11]. POT1, in a complex with TPP1, binds the $3^{\prime}$ single-stranded overhang in a sequence-specific manner $[12,13]$. POT1 and TPP1 function together by forming a stable heterodimer that pro- 
tects chromosome ends and regulates telomerase activity [14]. TIN2 simultaneously interacts with TRF1, TRF2, and TPP1, thus serving as an interaction hub of the shelterin complex $[15,16]$.

A shelterin-like complex has been identified in fission yeast Schizosaccharomyces pombe, suggesting that the telomere-end-protection mechanism by a shelterin-like complex is conserved [17]. The $S$. pombe shelterin complex is also composed of six proteins - Taz1, Rap1, Poz1, Tpz1, Pot1, and Ccq1 [17, 18]. Taz1 binds directly to the double-stranded telomeric repeats and is structurally and functionally similar to mammalian TRF1 and TRF2 [19]. Taz1 recruits Rap1, a homolog of mammalian RAP1, to telomeres [20-22]. The single-stranded overhang-binding proteins Pot1 and Tpzl are the respective homologs of mammalian POT1 and TPP1 [18, 23, 24]. Poz1, a possible homolog of mammalian TIN2, interacts with both Rap1 and Tpz1, thereby acting as a molecular bridge connecting the proteins bound to the single- and double-stranded regions of telomeres [18, 25, 26]. In addition, Ccq1 that is absent in mammalian shelterin directly interacts with Tpzl and plays a key role in recruiting telomerase to telomeres [27, 28].

Deletion of $\mathrm{tazl}^{+}, \mathrm{rapl}^{+}$, or $\mathrm{pozl}^{+}$leads to massive elongation of telomeres, suggesting that these proteins function as negative regulators of telomere length [18-20, 22]. Deletion of $\operatorname{tazl}^{+}$or $\mathrm{rapl}^{+}$also causes chromosome end fusions when cells are arrested in G1 phase by nitrogen starvation, underscoring their roles in chromosome end capping $[21,29]$. The Pot1-Tpzl heterodimer is crucial for telomere end protection, as deletion of either pot $1^{+}$or $t p z 1^{+}$results in rapid telomere loss $[18,23]$. Together with Ccq1, the Pot1-Tpz1 heterodimer also plays a key role in telomerase recruitment [18]. Ccq1 mediates the recruitment of telomerase to telomeres through a Rad3/Tel1-dependent interaction with the telomerase regulatory subunit Est1 [27, 30]. Notably, although $c c q 1 \Delta$ cells are only partially defective in telomere protection, the simultaneous deletion of the $c c q 1^{+}$and $\mathrm{pozl}^{+}$ genes leads to a severe telomere deprotection phenotype reminiscent of tpz $1 \Delta$ and pot $1 \Delta$ cells, suggesting that Ccq1 and Pozl play redundant roles in end protection [18]. However, the mechanism of why Ccq1 and Poz1 are redundantly required to prevent telomere fusions remains to be established. The $S$. pombe shelterin is also required for the maintenance of telomeric heterochromatin structure [2]. Ccq1 directly interacts with the Clr3 subunit of the Snf2/HDAC-containing repressor complex (SHREC), a regulator of heterochromatic gene silencing, to facilitate heterochromatin formation at telomeres [18, 31]. Mutations of Taz1, Rap1, Poz1, Tpz1, or Ccq1 that break the connections among shelterin subunits all result in defects in gene silencing at telomeres $[17,18,25,32]$.

In the past decade, structural studies of shelterin subunits have provided valuable insight into the structural and functional significance of shelterin proteins in telomere maintenance and protection. The POT1-TPP1 heterodimer (Pot1-Tpz1 in fission yeast) and the TRF homology (TRFH) domain-containing proteins (TRF1 and TRF2 in humans and Taz1 in fission yeast) use evolutionarily conserved oligonucleotide/oligosaccharide-binding (OB) folds and the Myb domains, respectively, to bind to single-stranded and double-stranded telomeric DNAs to protect chromosome ends from being recognized as DNA damage sites $[12,14,24,33-35]$. The C-terminal RCT domain of RAP1 is another evolutionarily conserved motif that mediates the interaction with the double-stranded telomeric DNA-binding protein (TRF2 in humans and Taz1 in fission yeast) for chromosome end protection [29]. In addition, our previous work also revealed that the TRFH domains of TRF1 and TRF2 function as telomeric protein docking sites that recruit different shelterin-associated factors with distinct functions to the chromosome ends [36]. Despite this progress, knowledge of the structure of the central hub of shelterin (TIN2 in humans and Poz1 in fission yeast) and how it functions as a bridge between the single- and double-stranded regions of telomeres still remain unknown.

Here, we report the crystal structure of the $S$. pombe Pozl complexed with two Poz1-binding motifs of Tpz1 and Rap1, which reveals that Poz1 adopts a dimeric conformation. Structure-based mutational analyses reveal that proper connection between Poz1 and Rap1 is required for the maintenance of telomere length homeostasis and telomere heterochromatin structure. In addition, the Poz1-Rap1 interaction is also required for telomere maintenance mediated by homologous recombination (HR) when Ccq1 is absent from telomeres. Remarkably, comparative analysis reveals a close structural resemblance between Pozl and the TRFH domains of the $S$. pombe Taz1 and human TRF1 and TRF2 proteins, suggesting that they all belong to the same TRFH family of proteins and might have the same ancestor during evolution.

\section{Results and Discussion}

\section{Structural determination of the Poz1-Tpzl complex}

Understanding how Poz1 functions as the hub of the shelterin complex connecting the double- and single-stranded regions of telomeres requires structural insight into the interactions between Poz1, Tpz1, and Rap1. We characterized the Poz1-Tpz1 interaction by yeast two-hybrid analysis (Supplementary information, 
A

Tpz1

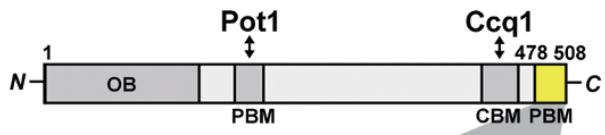

Poz1

Rap1

\begin{tabular}{|c|c|c|c|c|}
\hline 1 & & & 467491 & 693 \\
\hline BRCT & MYB & MYB & & RCT $-C$ \\
\hline
\end{tabular}

SpTpz1 478 ACEMCRLGLP HGSFFELLDWKKIEEFRNKS

ScTpz1 481 TETPVERSRSENAFLTFYQDWMRIARHRTDK

SoTpz1 296 AQT L LETDSENAFLTFYKDWIRIAKHRKEK

STTpz1

474 KTKQQLMDICEGEFHDLHKNWLRIKYRERM

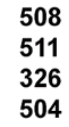

C
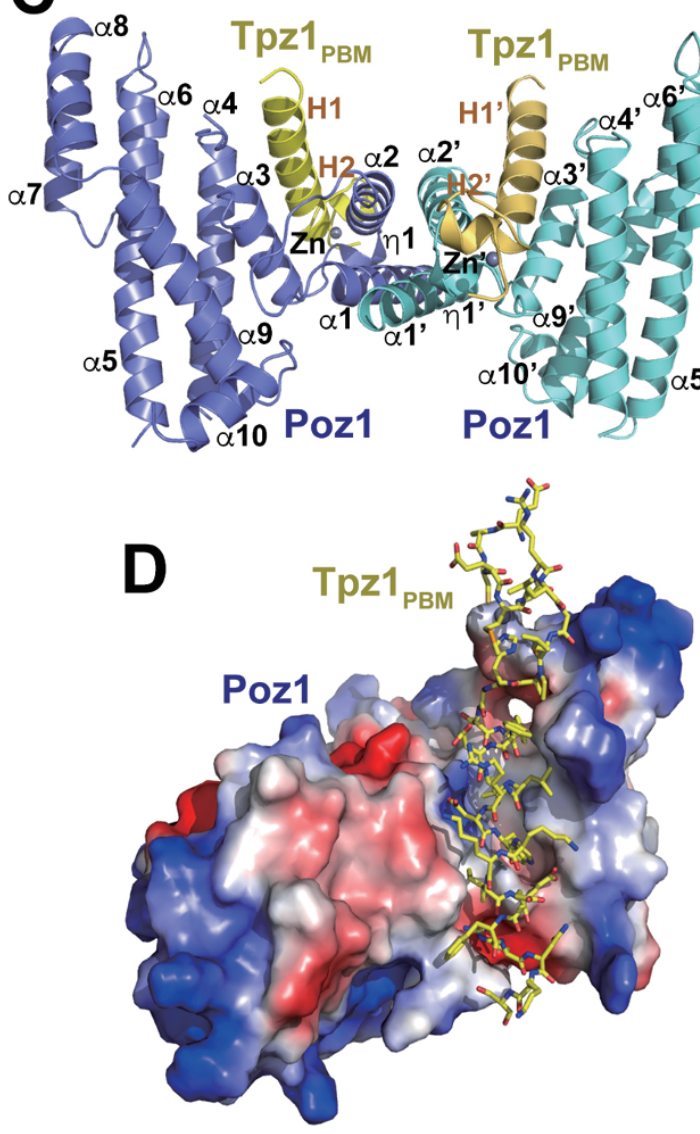

Tpz1 ${ }_{\text {PBM }}$

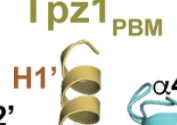

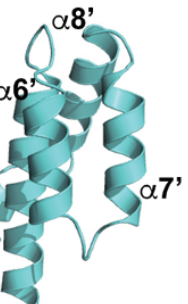
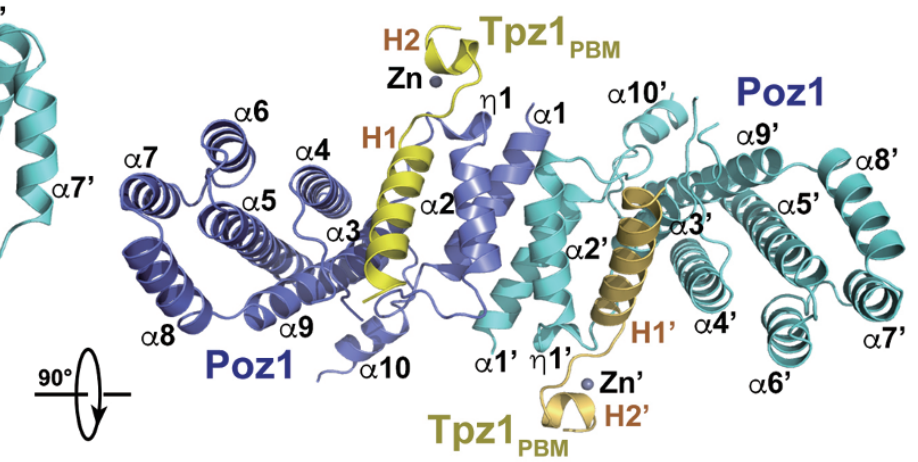

E

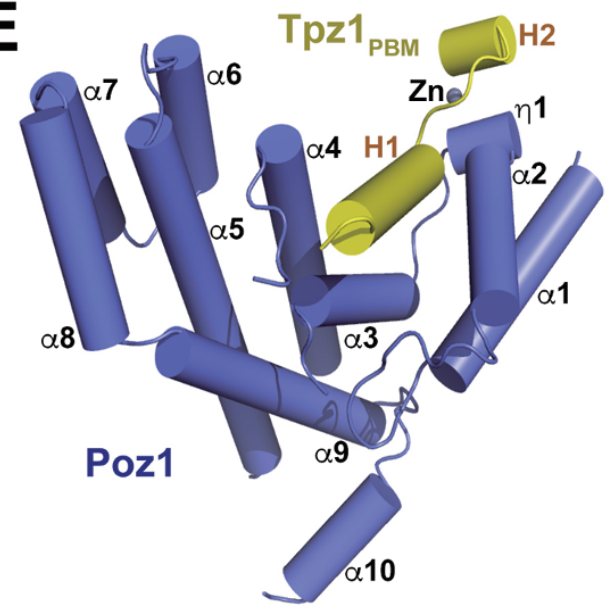

Figure 1 Overview of the Poz1-Tpz1 $1_{\mathrm{PBM}}$ complex structure. (A) Domain organization of the S. pombe Rap1-Poz1-Tpz1 subcomplex. Conserved domains, motifs, and interacting proteins are denoted. Poz1, Poz1-binding motif (PBM) of Tpz1, and Poz1-binding motif (PBM) of Rap1 are colored in slate blue, yellow, and magenta, respectively. The shaded areas indicate the Poz1-Rap1 and Poz1-Tpz1 interactions. (B) Sequence alignment of S. pombe Tpz1 $1_{\mathrm{PBM}}$ and its homologues. Conserved residues of Tpz1 $1_{\text {РвM }}$ are boxed and highlighted in red. S. pombe, NP_593908.2; S. cryophilus, XP_013021465.1; S. octosporus, XP_013017710.1; S. japonicus, XP_002172628.1. (C) Overall structure of the Poz1-Tpz1 $1_{\text {PBM }}$ complex in two orthogonal views. Poz1 is colored in slate blue and cyan, and Tpz $1_{\text {PBM }}$ in yellow and orange, respectively. (D) Electrostatic surface poten-

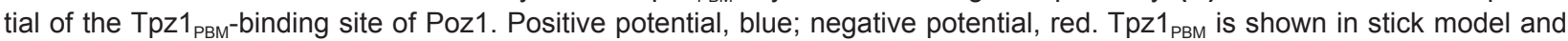
colored in yellow. (E) Ribbon diagram of the Poz1-Tpz1 $1_{\mathrm{PBM}}$ interaction. Poz1 is colored in slate blue and Tpz1 $1_{\mathrm{PBM}}$ in yellow. The secondary structure elements are labeled. 
Figure S1). Consistent with previous studies, we found that a short and highly conserved fragment of Tpzl (residues 478-508) is necessary and sufficient for binding to Poz1 [26, 37] (Supplementary information, Figure S1). Hereafter, we will refer to Tpz1 $1_{478-508}$ as the Poz1-binding motif of Tpz1 (Tpz1 $1_{\mathrm{PBM}}$ ) (Figure 1A and 1B).

An initial attempt to express full-length Pozl alone only yielded insoluble protein product (data not shown). In contrast, co-expression of Poz1 with Tpz $1_{\mathrm{PBM}}$ resulted in well-behaved Poz1-Tpz $1_{\mathrm{PBM}}$ binary complex, indicating that the correct folding of Pozl depends on the presence of $\mathrm{Tpz}_{\mathrm{PBM}}$ (Supplementary information, Figure
S2). Crystallization trials of the Poz1-Tpz1 $1_{\mathrm{PBM}}$ complex generated crystals that only diffracted to $\sim 4.5 \AA$ resolution (data not shown). After exhaustive optimization of the Pozl construct, a deletion mutant of Pozl that lacks residues 71-83 yielded proteins of good quality that were suitable for structural studies. Multiple sequence alignment of Pozl proteins from various species revealed that this region of Poz1 is highly variable in sequence (Supplementary information, Figure S3). Hereafter, for simplicity we will refer to Poz1 $\Delta 71-83$ as Pozl, unless stated otherwise. We determined the Poz1-Tpz $1_{\mathrm{PBM}}$ binary complex structure using the single-wavelength anomalous

Table 1 Crystal data collection and refinement statistics

\begin{tabular}{|c|c|c|}
\hline & Poz-Tpz1 $_{\text {PBM }}($ SeMet-SAD) & Rap1 $_{\mathrm{PBM}}-$ Poz1-Tpz1 ${ }_{\mathrm{PBM}}$ (Native) \\
\hline \multicolumn{3}{|l|}{ Data collection } \\
\hline Wavelength $(\AA)$ & 0.97860 & 0.97861 \\
\hline \multicolumn{3}{|l|}{ Cell dimensions } \\
\hline $\mathrm{a}, \mathrm{b}, \mathrm{c}(\AA)$ & $95.6,97.6,107.3$ & $81.2,85.7,116.0$ \\
\hline$R_{\text {merge }}(\%)$ & $5.9(67.1)^{*}$ & $10.1(73.2)^{*}$ \\
\hline$I / \sigma I$ & $21.3(2.4)^{*}$ & $17.3(2.5)^{*}$ \\
\hline Completeness (\%) & $99.7(99.8)^{*}$ & $100.0(100.0)^{*}$ \\
\hline Redundancy & $4.4(4.5)^{*}$ & $6.5(6.2)^{*}$ \\
\hline No. of reflections & 32723 & 15007 \\
\hline$R_{\text {work }} / R_{\text {free }}(\%)$ & $20.9 / 25.6$ & $25.0 / 30.2$ \\
\hline \multicolumn{3}{|l|}{ No. of atoms } \\
\hline Protein & 4280 & 4572 \\
\hline Zinc & 2 & 2 \\
\hline Sulfate & 50 & - \\
\hline Water & 227 & 11 \\
\hline \multicolumn{3}{|l|}{ B-factors $\left(\AA^{2}\right)$} \\
\hline Protein & 50.0 & 71.9 \\
\hline \multicolumn{3}{|l|}{ Ramanchandran plot } \\
\hline Favored region & $97.6 \%$ & $96.4 \%$ \\
\hline Allowed region & $100.0 \%$ & $100.0 \%$ \\
\hline Outlier region & $0.0 \%$ & $0.0 \%$ \\
\hline
\end{tabular}

*Highest resolution shell is shown in parenthesis. 
dispersion (SAD) method at a resolution of $2.5 \AA$ (Table 1; Supplementary information, Figure S4). The calculated electron density map allowed the unambiguous tracing of most of the complex except for an 11-residue disordered loop in Pozl (residues 117-127). The complex structure has been refined to an $R$-value of $20.9 \%\left(R_{\text {free }}=25.6 \%\right)$ with good geometry (Table 1).

\section{Overall structure of the Poz1-Tpz $1_{P B M}$ complex}

Unexpectedly, the Poz1-Tpz $1_{\mathrm{PBM}}$ complex structure reveals a 2:2 stoichiometry between Poz1 and Tpz1 $1_{\mathrm{PBM}}$ (Figure 1C). The N-terminal two $\alpha$ helices ( $\alpha 1$ and $\alpha 2)$ from both Poz1 monomers form a tightly packed four-helix bundle (Figure 1C), burying a total of $\sim 1020 \AA^{2}$ solvent accessible surface area, which is substantially larger than other crystal packing contacts. This observation strongly implies that the dimeric conformation observed in the crystals is unlikely to be the result of lattice packing. Experiments using calibrated gel-filtration chromatography showed that the elution peak of the Poz1-Tpz1 $1_{\mathrm{PBM}}$ complex corresponded to a molecular weight of $\sim 67 \mathrm{kDa}$ (Supplementary information, Figure S2), as would be expected if the dimeric interaction observed in the crystals is present in solution. This result corroborated our crystallographic finding and confirmed that Pozl indeed exists as a dimer in solution.

The Poz1-Tpz $1_{\mathrm{PBM}}$ complex exhibits a twisted butterfly-shaped structure measuring linear dimensions of $\sim 87$ $\AA \times 60 \AA \times 51 \AA$ (Figure 1C). Each Tpz1 $1_{\text {PBM }}$ polypeptide binds into a deep groove formed by only one monomer of Poz1, which does not overlap with the Poz1 dimeric interface (Figure 1C). Tpz $1_{\mathrm{PBM}}$ and Poz1 interact mainly through complementary hydrophobic surfaces (Figure 1D). The formation of the Poz1-Tpz $1_{\mathrm{PBM}}$ binary complex results in the burial of $\sim 1220 \AA^{2}$ of surface area at the interface. The Poz1 monomer consists of $10 \alpha$ helices, forming an elongated helix bundle (Figure 1E). Helices $\alpha 3$ to $\alpha 9$ constitute the core of the Poz1 monomer, in which two pairs of helices ( $\alpha 4$ and $\alpha 5, \alpha 6$ and $\alpha 7$ ) tightly pack against helices $\alpha 3, \alpha 8$, and $\alpha 9$ (Figure 1E). In contrast, helices $\alpha 1, \alpha 2$, and $\alpha 10$ are loosely connected to the helical core, forming two protrusions from the Nand C-termini of Poz1, respectively (Figure 1E). The large open wedge between helices $\alpha 1-\alpha 2$ and $\alpha 3-\alpha 4$ forms the binding groove for $\mathrm{Tpz}_{\mathrm{PBM}}$ (Figure 1E). Helix $\alpha 10$ folds back and packs almost perpendicularly to helix $\alpha 9$, forming a smaller cavity for the binding interface for Rap1 (see below) (Figure 1E).

\section{The dimeric interface of Pozl}

At the Poz1 dimeric interface, helices $\alpha 1$ and $\alpha 2$ pack together forming a symmetric antiparallel four-helix bundle with the two-fold symmetry perpendicular to the helix bundle axis (Figure 2A). The hydrophobic packing interface in the bundle is extensive, consisting of interdigitating residues from the helices (Val10, Thr13, and Phe17 of $\alpha 1$, and Ile33, Ala36, and Tyr40 of $\alpha 2$ ) (Figure 2B). Although the dimeric interface is predominantly hydrophobic, intermolecular electrostatic interactions provide additional specificity and stability to the dimer. At both ends of the helix bundle, two identical electrostatic networks, formed by Glu30 from one monomer and Glu3, Arg6, and Tyr40 from the other, seal both ends of the interface (Figure 2B). In addition, at one side of the bundle, Ser9 and Thr13 from both monomers contribute additional three hydrogen-bonding interactions at the Poz1 dimeric interface (Figure 2B).

To confirm the significance of the dimeric contacts observed in the crystal structure, we generated five missense mutations of residues at the Poz1 dimeric interface. All mutant proteins were co-expressed and purified with $T \mathrm{pz} 1_{\mathrm{PBM}}$ and the oligomeric states of these proteins were individually analyzed by gel-filtration chromatography (Figure 2C). Consistent with the structure, substitution of Phe17, Ile33, Ala36, and Tyr40 at the Pozl hydrophobic interface with a positively charged and bulky arginine residue completely disrupted the dimeric state of the Poz1-Tpz $1_{\mathrm{PBM}}$ complex; the elution profiles of these four mutants shifted toward the monomer species on gel-filtration chromatography (Figure 2C). Notably, the T13A mutant had an elution peak similar to the wild-type Poz1-Tpz $1_{\mathrm{PBM}}$ complex, suggesting that this mutant did not disrupt the dimeric interface (Figure 2C). Taken together, we therefore conclude that hydrophobic contacts are the major driving force for the dimer formation of Poz1.

\section{The Poz1-Tpz $1_{P B M}$ interface}

In the Poz1-Tpz $1_{\mathrm{PBM}}$ complex structure, the two Tpz1 $1_{\mathrm{PBM}}$ polypeptides adopt symmetric conformations and each Tpz1 $1_{\mathrm{PBM}}$ interacts with one Pozl molecule in the dimer (Figure 1C). Tpz1 $1_{\mathrm{PBM}}$ contains two $\alpha$ helices (H1 and $\mathrm{H} 2$ ) that are separated by a five-residue loop (Figure 1C). The long helix, H1, consists of residues Ser490 to Asn506. The hydrophobic portion of the amphipathic H1 helix of Tpz1 $1_{\text {PBM }}$ packs against the hydrophobic floor of the deep groove formed by Poz1 helices $\alpha 2, \alpha 3$, and $\alpha 4$ and the loop between $\alpha 1$ and $\alpha 2$, accounting for most of the buried surface area (Figure 3A). Five hydrophobic residues of Tpz $1_{\mathrm{PBM}}$, Phe491, Leu494, Trp498, Ile501, and Phe504 make intimate van der Waals contacts with the Poz1 groove (Figure 3A). In addition, nine hydrogen-bonding and ion-pair interactions mediated by Asp497, Lys500, Glu502, and Arg505 at the periphery of helix H1 of 

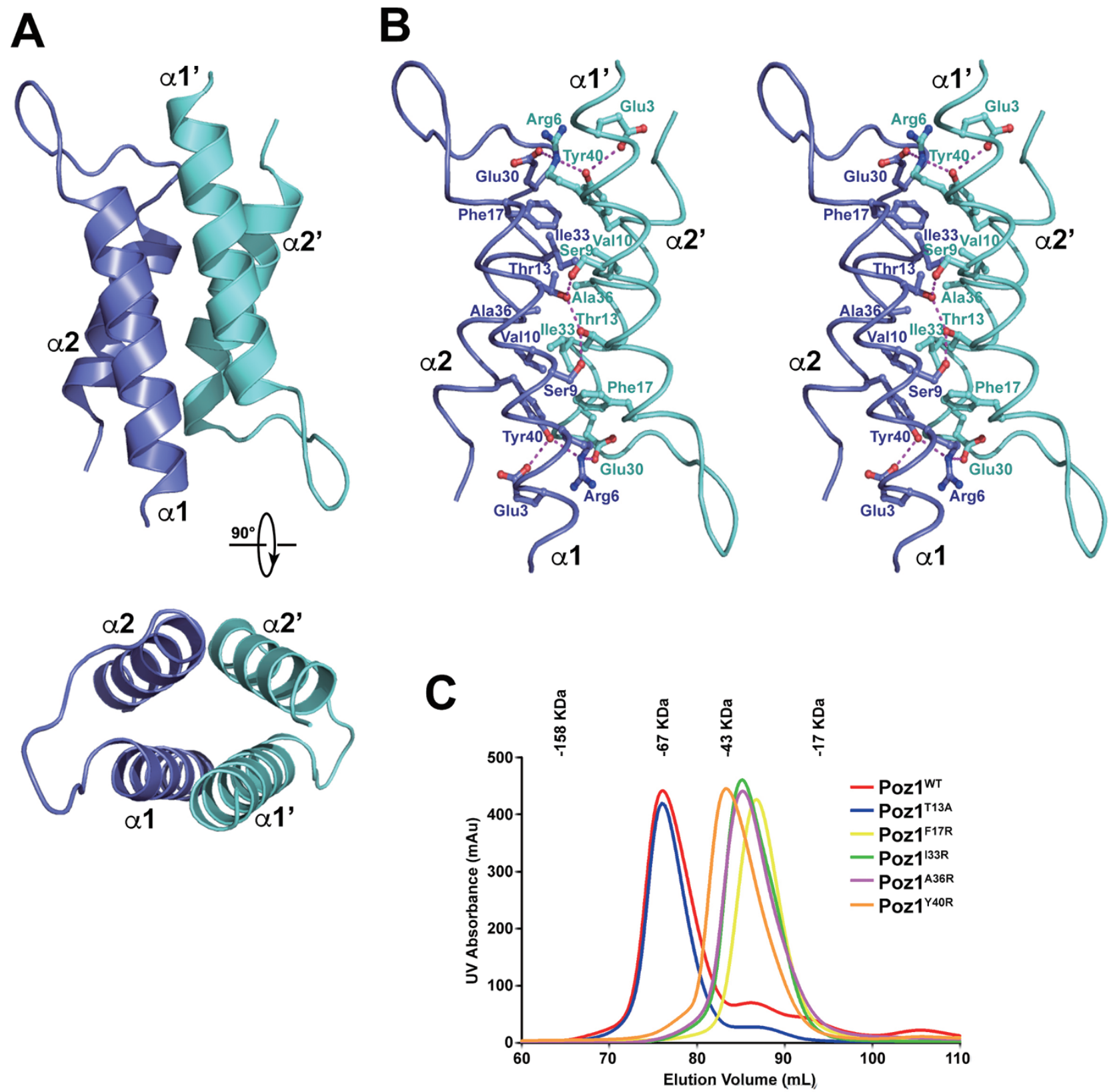

Figure 2 The dimeric interface of Poz1. (A) Ribbon diagram of the two $\alpha$ helices involved in dimer formation in two orthogonal views. Helices $\alpha 1$ and $\alpha 2$ of one Poz1 molecule are colored in slate blue, and $\alpha 1^{\prime}$ and $\alpha 2$ ' of the other in cyan. (B) Stereo view of the Poz1 dimeric interface. The Poz1 molecules are shown in ribbon representation and have the same color scheme as in (A). Residues important for the interaction are shown in ball-and-stick models and hydrogen bonding interactions are denoted by magenta dashed lines. (C) Poz1 is a dimer in solution. Gel-filtration chromatography profiles of wild-type and mutant Poz1-Tpz $1_{\mathrm{PBM}}$ are superimposed in different colors. Elution positions of the 158, 67, 43, and 17 kDa protein markers are indicated.

Tpz1 $1_{\mathrm{PBM}}$ further stabilize the relative positioning of the Tpz $1_{\mathrm{PBM}} \mathrm{H} 1$ helix on Poz1 (Figure 3B). The N-terminus of $\mathrm{Tpz} 1_{\mathrm{PBM}}$ including the short helix $\mathrm{H} 2$ protrudes outside of the major Poz1-Tpz1 $1_{\mathrm{PBM}}$ interface to make direct contacts with the loop between helices $\alpha 2$ and $\alpha 3$ of Pozl (Figure
3C). Notably, in this region of the complex, a zinc ion lies between Poz1 and Tpz1 $1_{\mathrm{PBM}}$, which is coordinated by two cysteine residues and two histidine residues contributed by both Poz1 (His49) and Tpz1 $1_{\text {РвM }}$ (Cys479, Cys482, and His488) (Figure 3C). This zinc ion helps further stabilize 

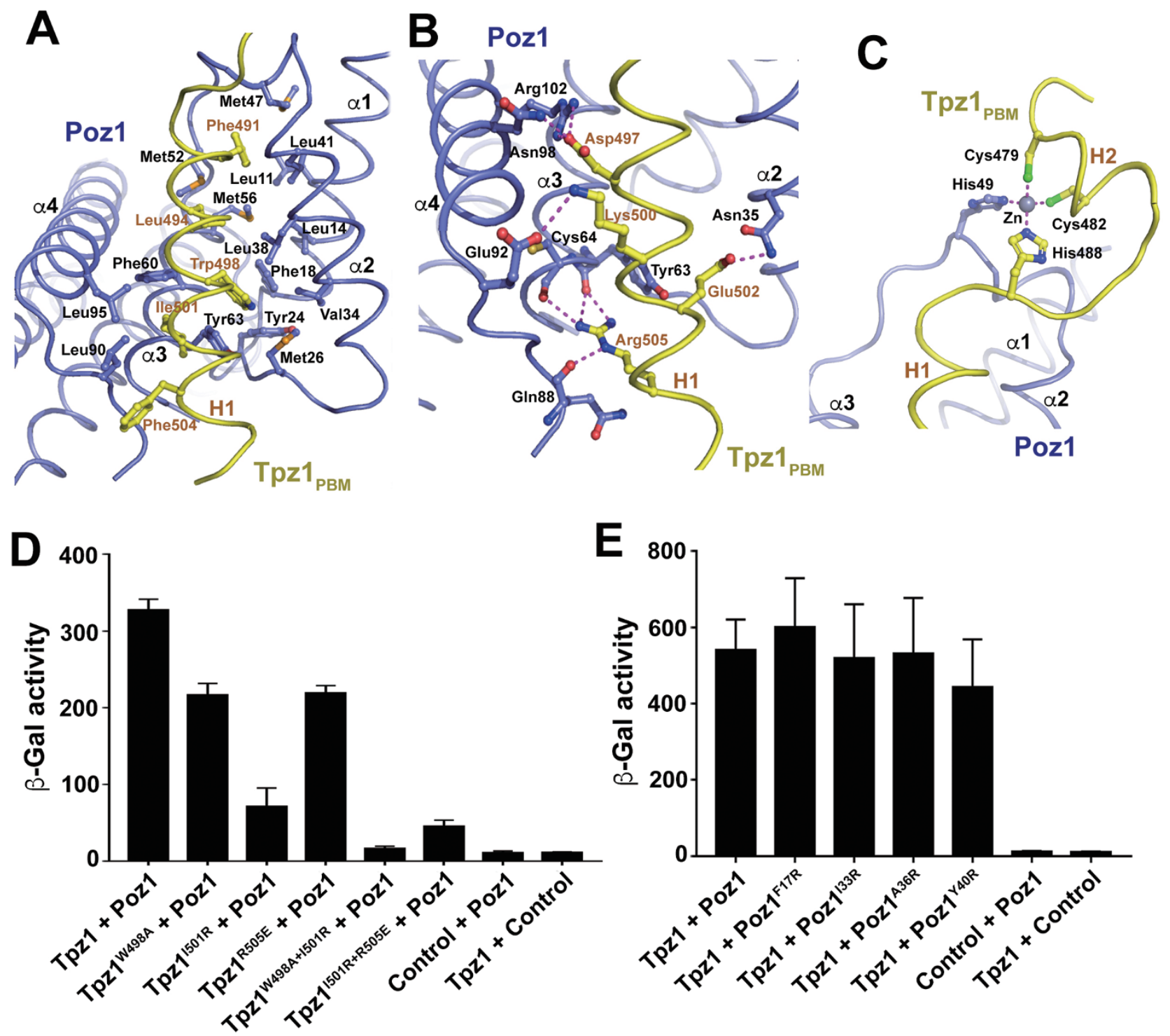

Figure 3 Structural analysis of the Poz1-Tpz1 $1_{\mathrm{PBM}}$ interface. (A) Hydrophobic interactions between Poz1 and Tpz1 $1_{\mathrm{PBM}}$. Interacting residues of Poz1 and Tpz1 $1_{\mathrm{PBM}}$ are shown in ball-and-stick models. (B) Electrostatic interactions between Poz1 and Tpz$1_{\mathrm{PBM}}$. Salt bridges and hydrogen bonding interactions are denoted by magenta dashed lines. (C) Zinc-finger structure formed by Poz1 and Tpz1 $1_{\mathrm{PBM}}$. The zinc ion is shown as a dark grey solid sphere. Four residues (His49 of Poz1 and Cys479, Cys482 and His488 of Tpz1 $1_{\mathrm{PBM}}$ ) forming the zinc finger are shown in ball-and-stick models. (D) Effects of mutations of Tpz1 on the interaction between Poz1 and Tpz1 analyzed by a yeast two-hybrid assay. Data are average of three independent $\beta$-galactosidase measurements. Error bars in the graph represent standard deviation. (E) Mutations of Poz1 with defects in dimer formation have no effect on the interaction between Poz1 and Tpz1.

the interaction between Poz1 and Tpz $1_{\mathrm{PBM}}$.

To corroborate our structural analysis, we next examined whether missense mutations of key residues at the interface could weaken or disrupt the interaction between Pozl and Tpz1. At the center of this interface, the side chains of Tpz1 Trp498 and Ile501 fit into two adjacent hydrophobic pockets in the Tpz1-binding groove of Poz1 (Figure 3A). We found that the Tpzl mutation I501R greatly weakened the Poz1-Tpz1 interaction [26, 37] (Figure 3D). However, substitution of Tpz1 Trp498 with alanine only partially weakened the interaction with Poz1, suggesting that this large hydrophobic side chain contributes to but is not essential for the interaction (Figure 3D). Similarly, disruption of electrostatic interactions between Poz1 and Tpz1 at the periphery of the interface by the Tpz1 mutation R505E also only partially 
weakened the interaction between Poz1 and Tpz1 (Figure 3D). Notably, combination of Tpz1 mutation I501R with either W498A or R505E abolished the interaction with Pozl (Figure 3D). Collectively, we conclude that both the hydrophobic and the electrostatic contacts observed in the crystal structure are important for the interaction between Pozl and Tpzl.

Close inspection of the Poz1-Tpz $1_{\mathrm{PBM}}$ complex structure revealed that the Poz1-Tpz1 interface and the Poz1 dimerization interface do not overlap. This observation promoted us to examine the role of Pozl dimerization in Tpz1 binding. As shown in Figure 2C, the four monomeric mutants of Poz1 (F17R, I33R, A36R, and Y40R) all exhibited wild-type interaction with $\mathrm{Tpz} 1_{\mathrm{PBM}}$ (Figure 3E; Supplementary information, Figure S5). Therefore, we conclude that Pozl dimerization is not a prerequisite for the stable association of Pozl and Tpz1.

\section{Crystal structure of the Rap $1_{P B M}-P o z 1-T p z 1_{P B M}$ complex}

To gain insight into the molecular basis of how Pozl recognizes Rap1, we characterized the Poz1-Rap1 interaction using yeast two-hybrid analysis. Consistent with previous studies, our data revealed that a short and highly conserved fragment of Rap1 consisting of residues 466491 was necessary and sufficient for binding Poz1 [25] (Figure 4A and 4B). Hereafter, we will refer to Rap $1_{466-491}$ as Rap $1_{\mathrm{PB}}$ (Poz1-binding motif). The equilibrium dissociation constant $\left(K_{\mathrm{d}}\right)$ between the Rap $1_{\mathrm{PBM}}$ peptide and the Poz1-Tpz $1_{\text {PBM }}$ complex was $\sim 1.8 \mu \mathrm{M}$, which was not strong enough to support the formation of a stable Rap$1_{\mathrm{PBM}}-$ Poz1-Tpz $1_{\mathrm{PBM}}$ ternary complex in solution (Supplementary information, Figure S6). To unravel the structural basis of the interaction between Poz1 and Rap $1_{\mathrm{PBM}}$, we fused the Rap $1_{\text {PBM }}$ peptide to the C-terminus of Poz1 and co-expressed this fusion protein with $\mathrm{Tpz} 1_{\mathrm{PBM}}$. We crystallized the Rap $1_{\mathrm{PBM}^{-}}-\mathrm{Poz} 1-\mathrm{Tpz} 1_{\mathrm{PBM}}$ complex and determined its structure by molecular replacement at a resolution of $3.1 \AA$ (Figure $4 \mathrm{C}$; Table 1). The complex structure was refined to an $R$-value of $25.0 \%\left(R_{\text {free }}=30.2 \%\right)$ with good geometry (Table 1). The calculated electron density map shows that residues 466-482 of Rap $1_{\mathrm{PBM}}$ assume a well-defined conformation (Supplementary information, Figure S7), and the Poz1-Tpz1 $1_{\mathrm{PBM}}$ moiety exhibits essentially the same conformation as that in the Poz1-Tpz $1_{\mathrm{PBM}}$ binary complex structure (Figure 4C).

The structure of the Rap $1_{\mathrm{PBM}}-\mathrm{Poz} 1-\mathrm{Tpz} 1_{\mathrm{PBM}}$ complex reveals that the Rap $1_{\mathrm{PBM}}$ polypeptide has an irregular conformation that meanders along the surface of helices $\alpha 5$ and $\alpha 9$ of Poz1 opposite the Tpz $1_{\mathrm{PBM}}$-binding site (Figure 4C and 4D). The binding of Rap $1_{\mathrm{PBM}}$ to Poz1 results in the burial of $\sim 780 \AA^{2}$ of surface area at the interface. This is substantially smaller than that of the
Poz1-Tpz $1_{\mathrm{PBM}}$ interface, explaining the weaker interaction between Pozl and Rap $1_{\mathrm{PBM}}$. The structure of the complex reveals that both electrostatic and hydrophobic contacts play important roles in the interaction between

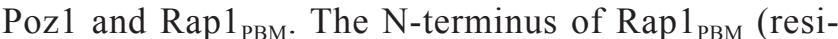
dues Asn466-Glu476) adopts a zig-zagged conformation stabilized by an extensive intermolecular electrostatic interaction network (Figure 4E). In particular, the long side chains of Glu136 and Arg218 of Poz1 not only contribute six electrostatic interactions to this network, but also determine the topography of the Pozl surface, which in turn defines the zig-zagged conformation of Rap $1_{\text {PBM }}$ (Figure 4E). Charge swapping mutations of these two residues completely abolished the interaction between Poz1 and Rap1, underscoring their importance in the recognition of Rap $1_{\mathrm{PBM}}$ by Pozl (Figure 4F). The zig-zagged conformation of Rap $1_{\mathrm{PBM}}$ positions the side chains of Rap $1_{\mathrm{PBM}}$ Ile470-Phe471-Val472 into a continuous hydrophobic cavity with a complementary surface, defining the recognition specificity of Rap $1_{\mathrm{PBM}}$ by Poz1 (Figure 4E). Notably, the hydrophobic cavity is partially formed by helix $\alpha 10$ of Poz1, which protrudes out from the helical core of Poz1 (Figure 4E). In addition, Leu478 and Ile480 of Rap1 also make hydrophobic contacts with Poz1 (Figure 4G). In support of the crystal structure, substitution of the conserved hydrophobic residues of Rap $1_{\mathrm{PBM}}$ (Phe471, Leu478, or Ile480) at the interface with a positively charged and bulkier arginine residue was sufficient to eliminate the interaction with Pozl in the yeast two-hybrid assay (Figure 4F). Therefore, we conclude that the hydrophobic interface between Poz1 and Rap $1_{\mathrm{PBM}}$ is necessary for the binding of Rap $1_{\mathrm{PBM}}$ to Poz1.

The C-terminal 9 residues of Rap $1_{\text {PBM }}$ (Leu483Asn491) are not visualized in the electron density map. This region of Rap $1_{\mathrm{PBM}}$ contains three acidic amino acids (Figure 4B). The surface of helices $\alpha 7$ and $\alpha 8$ of Poz1 that is very close to the last visible residue in Rap $1_{\mathrm{PBM}}$ (Leu482) has a highly positive potential (Figure 4D). Although the model of Rap $1_{\text {PBM }}$ cannot be extended unambiguously beyond Leu482, the close spatial disposition of the C-terminal acidic tail of Rap $1_{\mathrm{PBM}}$ and helices $\alpha 7$ and $\alpha 8$ of Poz1 suggests that these two regions are associated closely. To investigate the importance of this interface, we substituted Rap1 487-SDSE-490 with four alanine residues and examined the Poz1-Rap1 interaction using yeast two-hybrid analysis. As expected, the ' $4 \mathrm{~A}$ ' mutant greatly weakened the interaction between Rap1 and Poz1 (Figure 4F). Similarly, replacement of six basic residues of Poz1 in helices $\alpha 7$ and $\alpha 8$ (Lys180, Arg187, Lys190, Lys192, Arg194, and Lys196) with glutamate residues (' $6 \mathrm{E}$ ' mutant) on the Pozl side of the interface 


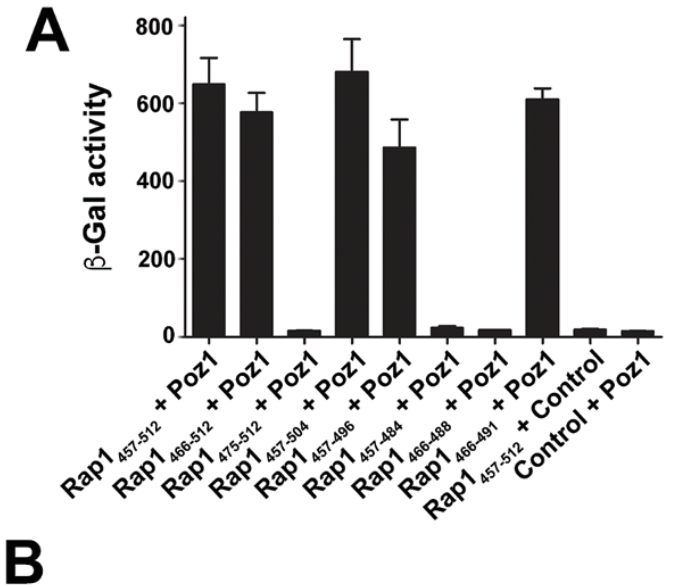

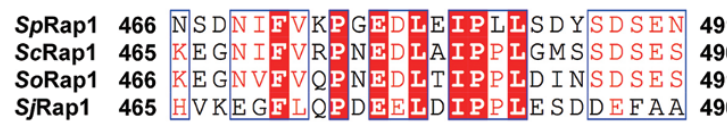
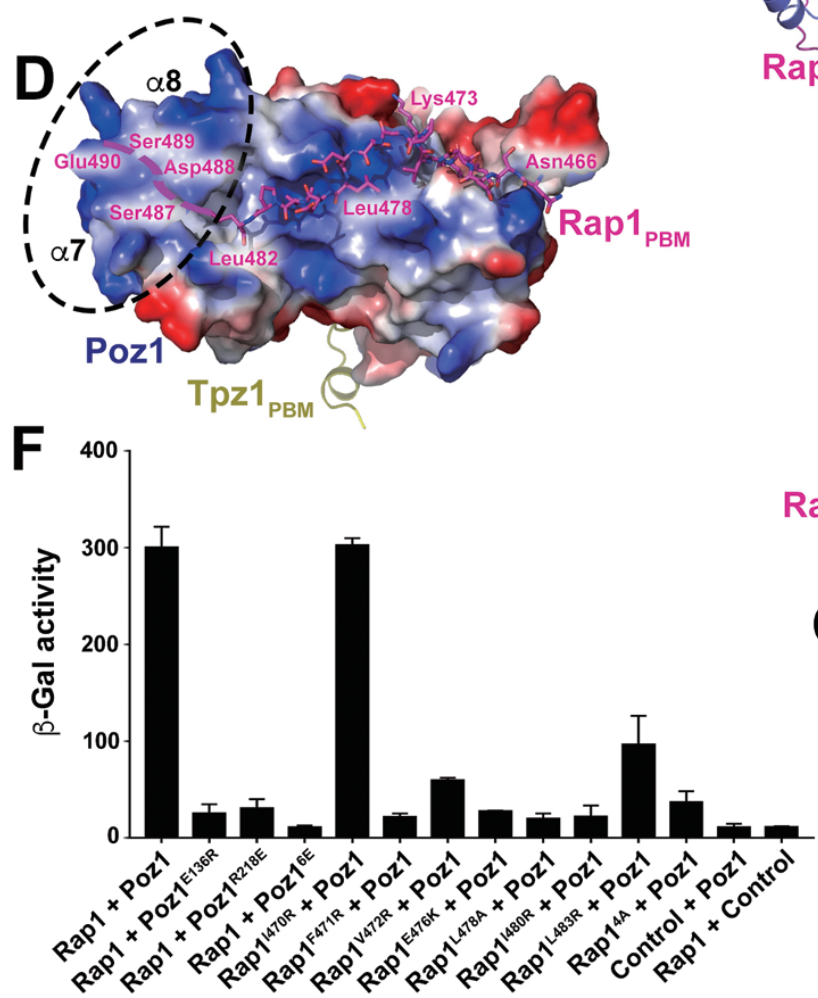
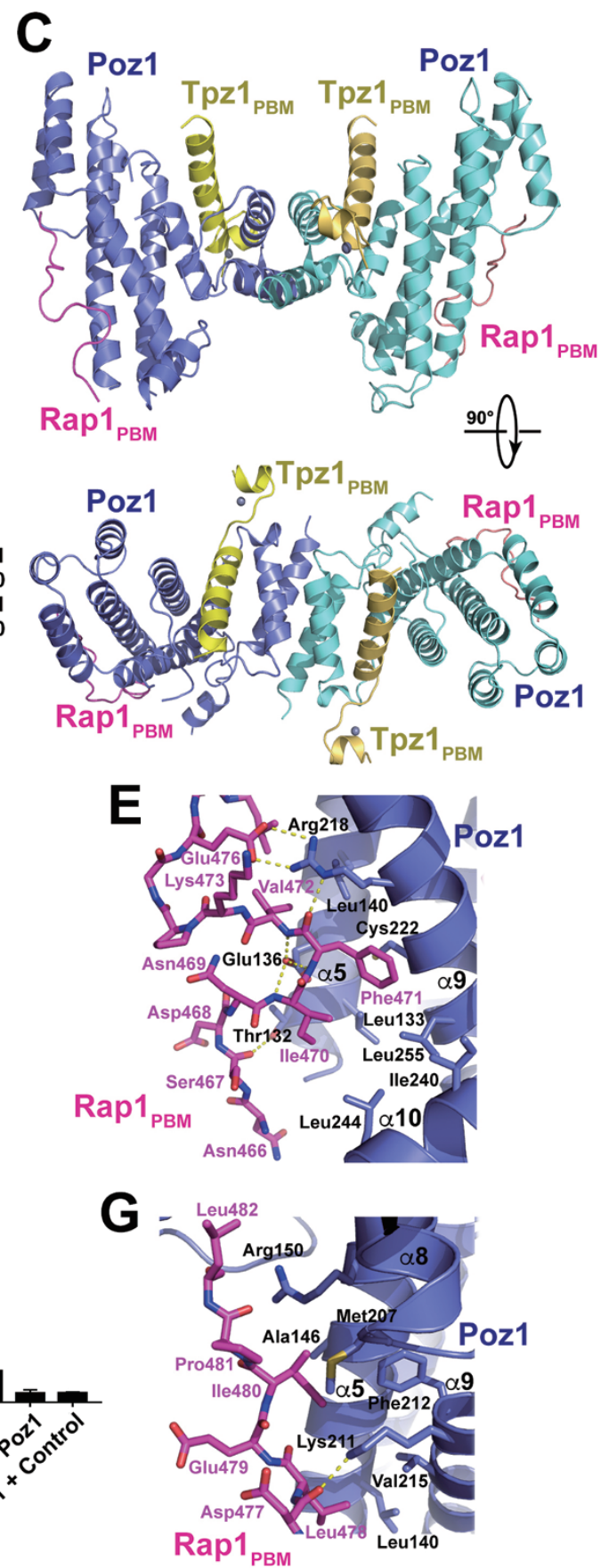

Figure 4 Structure of the Rap1 $1_{\mathrm{PBM}}-\mathrm{Poz1}-\mathrm{Tpz} 1_{\mathrm{PBM}}$ complex. (A) Characterization of the interaction between Rap1 and Poz1 by yeast two-hybrid assay. (B) Sequence alignment of the Poz1-binding motif (PBM) of S. pombe Rap1 and its homologues. Conserved residues of Rap1 $1_{\mathrm{PBM}}$ are boxed and highlighted in red. S. pombe, NP_596285.1; S. cryophilus, XP_013020902.1; S. octosporus, XP_013018075.1; S. japonicus, XP_002174258.2. (C) Overall structure of the Rap1 $1_{\mathrm{PBM}}-\mathrm{Poz}_{\mathrm{O}}-\mathrm{TpZ}_{\mathrm{PBM}} \mathrm{Complex}$ in two orthogonal views. The two Rap $1_{\mathrm{PBM}}$ molecules are colored in magenta and salmon, respectively. Poz1 and Tpz1 $1_{\mathrm{PBM}}$ have the same color scheme as in Figure 1C. (D) Electrostatic surface potential of the Rap $1_{\mathrm{PBM}}$-binding site of Poz1. Positive potential, blue; negative potential, red. Rap $1_{\mathrm{PBM}}$ is shown in stick model and Tpz1 $1_{\mathrm{PBM}}$ in ribbon model. Dashed ellipse denotes the basic area formed by $\alpha 7$ and $\alpha 8$ of Poz1, which might be involved in interacting with the C-terminal part of Rap1 ${ }_{\mathrm{PBM}}$ (487-

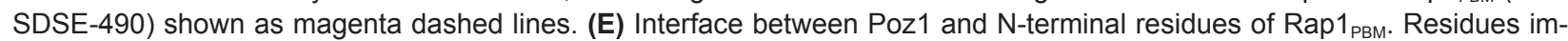
portant for the interaction are shown in stick models and hydrogen bonding interactions are denoted by yellow dashed lines. (F) Effects of the Rap1 and Poz1 mutations on the Rap1-Poz1 interaction analyzed by yeast two-hybrid assay. (G) Interface between Poz1 and C-terminal residues of Rap1 $1_{\mathrm{PBM}}$. The color scheme is the same as in (E). 
also disrupted the interaction (Figure 4F). Taken together, we conclude that the electrostatic interactions between the C-terminal acidic tail of Rap $1_{\mathrm{PBM}}$ and the Pozl basic patch on helices $\alpha 7$ and $\alpha 8$ are important for the interaction between Poz1 and Rap $1_{\mathrm{PBM}}$.

Functional analyses of the Poz1-Rapl interaction and Poz1 dimerization

Consistent with our structural data, previous studies showed that the same mutations of key interface residues of Tpz1 (W498A/I501R and I501R/R505E) that disrupted the Poz1-Tpzl interaction in our yeast two-hybrid assay caused telomere elongation but still protected telomeres against fusions. This suggests that the Poz1-Tpz1 interaction is important for telomere length homeostasis but not for telomere protection [26, 37] (Figure 3A, 3B, and $3 \mathrm{D})$. To further investigate the functional importance of the interface between Pozl and Rap1 in vivo, a series of mutations that are deficient in the Poz1-Rap1 interaction were studied for their effects on telomere length homeostasis, telomere protection, and maintenance of telomere heterochromatin structure. All the mutant proteins were expressed at near wild-type levels in yeast cells, suggesting that these mutations did not interfere with the protein stability (Supplementary information, Figure S8). Rap1 and Poz1 are key negative regulators of telomere extension by telomerase [18, 27, 37-39]. Consistent with published results, deletion of $\mathrm{poz}^{+}$or $\mathrm{rapl}^{+}$from yeast cells resulted in a dramatic increase in telomere length and length heterogeneity compared to wild-type cells [18-20, 22] (Figure 5A, lanes 2 and 3). Four point mutations (poz1 ${ }^{R 218 E}, \operatorname{rapl}^{\text {F471R }}, \operatorname{rapl}^{E 476 K}$, and $\operatorname{rapl}^{L 478 A}$ ) that abolished the Poz1-Rap1 interaction in the yeast two-hybrid assay displayed a significant loss of function in telomere length regulation and resulted in long and heterogeneous telomeres (Figure 5A, lanes 8-11). Notably, the rap $1^{4 A}$ mutant that retained partial Pozl-binding activity exhibited the least defect in suppressing telomere elongation (Figure 5A, lane 12). Collectively, these results suggest that the Poz1-Rap1 interaction plays a crucial role in telomere length regulation.

Poz1 and Rap1 are required for the maintenance of subtelomere heterochromatin structure, and consequently poz $1 \Delta$ and rap $1 \Delta$ deletion mutants display defects in gene silencing at subtelomeres $[19,20,25,40,41]$. To address the functional significance of the Tpz1-Poz1Rap1 complex in subtelomeric gene silencing, we ana-

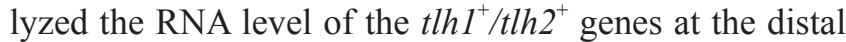
end of subtelomeres by reverse transcription-quantitative PCR [42]. The data clearly showed transcription of the $\operatorname{tlh} 1^{+} / t h 2^{+}$genes to be highly de-repressed in the mutant stains where the Poz1-Rap1 interaction was disrupted
(Figure 5B). These data indicate that the Poz1-Rap1 interaction is essential for the subtelomeric gene silencing.

To analyze how the Poz1-Rap1 interaction contributes to telomere end protection, we next examined non-homologous end joining (NHEJ)-dependent telomere end fusions of these mutants in G1 phase using pulsed field gel electrophoresis (PFGE) of NotI-digested chromosomal DNA followed by Southern blotting using telomere probes [43] (Figure 5C). Deletion of the $\mathrm{rapl}^{+}$gene resulted in telomere end fusion (Figure 5C, lane 3). This result is consistent with previous studies and suggests that Rap1 is essential for the prevention of the NHEJ-dependent fusion of telomere ends in G1 phase [20, 21]. In contrast, none of the mutations disrupting the Poz1-Rap1 interaction led to telomere fusions. Thus, the Poz1-Rap1 interaction is not required for telomere end protection (Figure 5C, lanes 8-12). Collectively, our mutational studies revealed that the Poz1-Rap1 interaction is essential for telomere length regulation and subtelomeric gene silencing but is dispensable for telomere end protection against fusion in $\mathrm{G} 1$ phase.

To examine the functional significance of the Poz1 dimerization, we carried out similar experiments to analyze four point mutations of Pozl that are deficient in dimerization (Figure 2C; Supplementary information, Figure S8). Surprisingly, none of these mutations caused any detectable defect in telomere length homeostasis, subtelomeric gene silencing, or telomere end protection (Figures 5A, 5B, and 5C), suggesting that Pozl dimerization is not required for these telomere functions when only Poz1 dimerization is disrupted in yeast cells.

Ccq1 is required for telomerase recruitment and inhibition of DNA damage-induced checkpoint activation at telomeres [18, 27, 28, 30, 44-46]. Deletion of $c c q 1^{+}$ results in progressive telomere shortening and activates a DNA damage checkpoint pathway. The shortened telomeres in $c c q 1 \Delta$ cells are maintained via $H R$, although a portion of unprotected chromosome ends are fused [18, 26, 37] (Figure 5D and 5E, lane 8). Notably, when $c c q 1^{+}$ and $\mathrm{poz} 1^{+}$were simultaneously deleted, $c c q 1 \Delta \mathrm{poz} 1 \Delta$ cells rapidly lost their telomeres and survived by forming self-circularized chromosomes, suggesting that Ccq 1 and Poz1 are redundantly required to prevent telomere fusions [18, 26, 37] (Figure 5D and 5E, lane 9). To investigate how Poz1 and Ccq1 cooperate in telomere maintenance and protection, we introduced the Rap1-binding deficient mutant poz $1^{R 218 E}$ and the Poz1 dimerization defective mutant poz $1^{A 36 R}$ into $c c q 1 \Delta$ cells and monitored telomere length and chromosome circularization by Southern blot and PFGE. As a control, the Pozl-binding deficient mutant $t p z 1^{I 501 R}$ was introduced into $c c q 1 \Delta$ cells and resulted in complete loss of telomeres and 

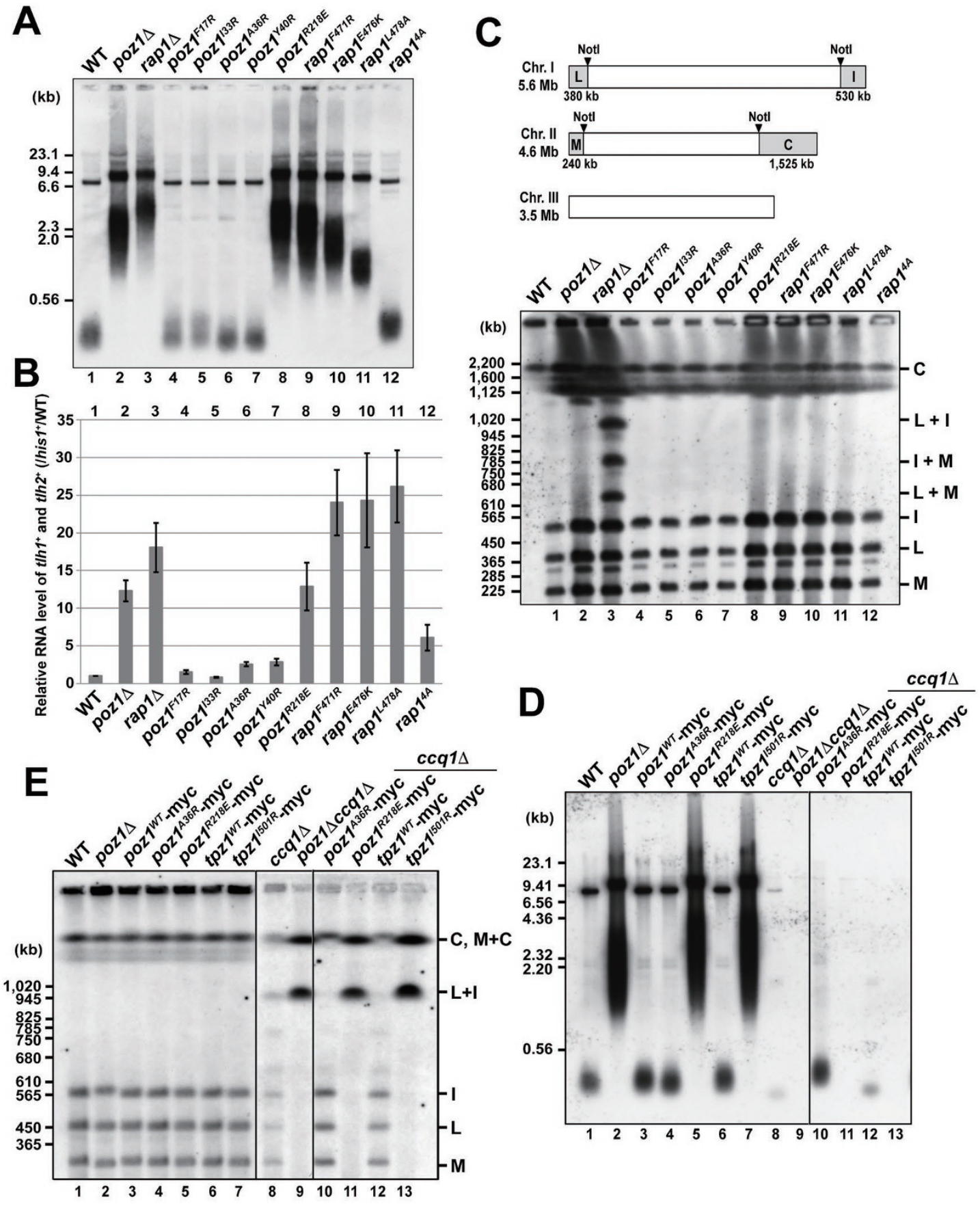

Figure 5 Functional analyses of the Poz1 dimer interface and the Poz1-Rap1 interaction. (A) Analysis of telomere length in various poz1 and rap1 mutants. Apal-digested genomic DNAs from indicated strains were subjected to Southern hybridization using the telomere repeats as the probe. (B) Telomere gene silencing. RNA expression level of subtelomeric $t / h^{+}$genes was analyzed by reverse transcription-quantitative PCR. The value of the $t / h^{+}$genes was normalized by that of the $h i s 1^{+}$gene. Bars and error bars indicate mean and SEM of three experiments. (C) Upper panel: schematic of Notl restriction sites on fission yeast genome. Lower panel: analysis of various poz1 and rap1 mutants for telomere protection. Cells were arrested in G1 phase by nitrogen starvation. Chromosomal DNAs were prepared in agarose plugs and separated by PFGE after Notl digestion. The chromosomal DNA was transferred to a nylon membrane and hybridized with a probe specific for telomere repeats. Southern blotting was performed using a probe mixture that recognizes Notl-digested chromosomal DNA fragments. Letters on the right hand side of the gel indicate the identities of Notl-digested chromosomal DNA fragments. (D) Analysis of telomere length in various mutants. Southern blotting was performed as in (A). (E) Analysis of various mutants for telomere protection. Cells were grown exponentially in YES. PFGE was performed as in (C). 
self-circularized chromosomes, a phenotype identical to

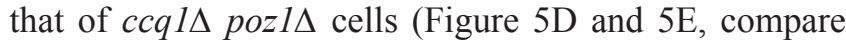
lanes 9 and 13). This is consistent with previous studies showing that the Poz1-Tpz1 interaction is required to recruit Poz1 to telomeres [26, 37]. Notably, the majority of $c c q 1 \Delta \mathrm{poz} 1^{R 218 E}$ cells died and rare survivor cells completely lost their telomeres and had self-circularized chromosomes (Figure 5D and 5E, lane 11). This result indicates that proper connection between single- and double-stranded telomeric DNAs mediated by the Poz1Rap1 interaction is required for the maintenance of the shortened telomeres in $c c q 1 \Delta$ cells. In contrast, $c c q 1 \Delta$ poz $1^{A 36 R}$ cells maintained stable telomeres with no circularized chromosomes (Figure 5D and 5E, lane 10). The stable telomere length in $c c q 1 \Delta$ poz $1^{A 36 R}$ cells is longer than that in ccq $1 \Delta$ cells (Figure 5D, compare lanes 8 and 10), suggestive of a possible role of Poz1 dimerization in suppressing HR-mediated telomere elongation in $c c q 1 \Delta$ cells.

In the shelterin complex, Tpzl functions as a molecular scaffold and simultaneously interacts with Poz1, Ccq1, and Pot1 [18]. Gel-filtration analysis showed that Poz1, Ccq1, Tpz1, and Pot1 form a stable quaternary complex (Supplementary information, Figure S9). A recent study reported that, in addition to Poz1, Ccq1 can also form a homodimer [47]. Our yeast two-hybrid analysis confirmed that the C-terminal coiled-coil domain of Ccq1 (residues 439-735) indeed mediates homodimerization interaction (Supplementary information, Figure S10). Therefore, both Poz1 and Ccq1 can mediate the dimeric conformation of the Ccq1-Tpz1-Pot1-Poz1 complex. Consistent with this idea, gel-filtration analysis showed that the single mutation Poz1 ${ }^{\mathrm{A} 36 \mathrm{R}}$ did not disrupt the dimeric state of the Ccq1-Tpz1-Pot1-Poz1 complex (Supplementary information, Figure S9). This is likely the reason why disruption of Poz1 dimerization alone did not cause any defect at telomeres (Figures 5A, 5B, and 5C). The functional significance of Pozl dimerization awaits further studies.

\section{Structural resemblance of Poz1 to other telomere pro- teins}

The $\alpha$-helical architecture of Poz1 prompted us to investigate the structural relationship between Pozl and other telomere proteins with domains of $\alpha$-helical architecture - the TRFH domains of human TRF1 and TRF2 and $S$. pombe Taz1 [36, 48, 49] (Figure 6A). Pairwise structural analysis revealed an unequivocal structural similarity of Poz1 to other TRFH domains (Supplementary information, Figure S11). Both Poz1 and the TRFH domains of TRF1 and TRF2 contain $10 \alpha$ helices. Moreover, the position of each helix in Pozl matches well to a corresponding helix in TRFH (Supplementary information, Figure S11). Despite a relatively low sequence conservation between Poz1 and the TRFH domains ( $\sim 5 \%$ identity between Poz1 and TRF $1_{\text {TRFH }}$ and $~ 8 \%$ identity between Poz1 and TRF $2_{\text {TRFH }}$ ), the Poz1 structure can be superimposed onto TRF $1_{\mathrm{TRFH}}$ and $\mathrm{TRF} 2_{\mathrm{TRFH}}$ with a rootmean-square deviation (r.m.s.d.) of 4.2 and $4.3 \AA$, respectively (Supplementary information, Figure S11). In addition to the overall structural similarity, Pozl and the TRFH domains of TRF1 and TRF2 share several specific features. Most notably, both Poz1 and the TRFH domains of TRF1 and TRF2 utilize the same concave groove to bind a short motif of their interacting partners (Figure 6A; Supplementary information, Figure S12). In addition, both Pozl and the TRFH domains also adopt similar homodimeric conformations in an antiparallel arrangement mainly through the first two helices of the proteins (Figure 6A). Collectively, these similarities support the notion that the structure of Pozl closely resembles those of the TRFH domains of TRF1 and TRF2.

The $S$. pombe homolog of human TRF1 and TRF2, Taz1, also contains an $\alpha$-helical domain that has been considered as the TRFH domain of Taz1 [10, 20, 50] (Figure 6A). Our previous structural studies confirmed that $\mathrm{Taz} 1_{\mathrm{TRFH}}$ indeed exhibits similar topological architecture to the TRFH domains of human TRF proteins [48] (Figure 6A). Consistently, pairwise structural comparison showed that Pozl is also structurally similar to Taz $1_{\mathrm{TRFH}}$ with an r.m.s.d. of $4.0 \AA$ in the positions of $139 \mathrm{C} \alpha$ atoms of equivalent residues (Supplementary information, Figure S11). In contrast to Poz1 and the TRFH domains of TRF1 and TRF2, Taz1 $1_{\text {TRFH }}$ adopts a monomeric conformation, the structural feature of helices $\alpha 1$ and $\alpha 2$ of Taz $1_{\text {TRFH }}$ does not support a dimeric interface [48] (Figure 6A). Nevertheless, our comparative analysis demonstrated that both mammalian TRF1 and TRF2 and $S$. pombe Pozl and Taz1 shelterin proteins contain an $\alpha$-helical TRFH domain.

Close inspection of the architecture of the $S$. pombe and human shelterin complexes suggested that Pozl functions as the central hub connecting the single- and double-stranded regions of the telomeres just as TIN2 does in the human shelterin complex (Figure 6B). Functionally, Poz1 and TIN2 define the boundary between the two sub-regions in shelterin that play distinct roles in telomere length regulation; Pot1-Tpz1 and POT1TPP1 are positive regulators of telomere length homeostasis, whereas Poz1-Rap1-Taz1 and TIN2-TRF1-TRF2 are negative regulators for this process $[3,14,18,51]$. Notably, primary sequence analysis predicted that TIN2 contains an N-terminal $\alpha$-helical domain (TIN2N) that shows some sequence similarity to Pozl (Supplemen- 


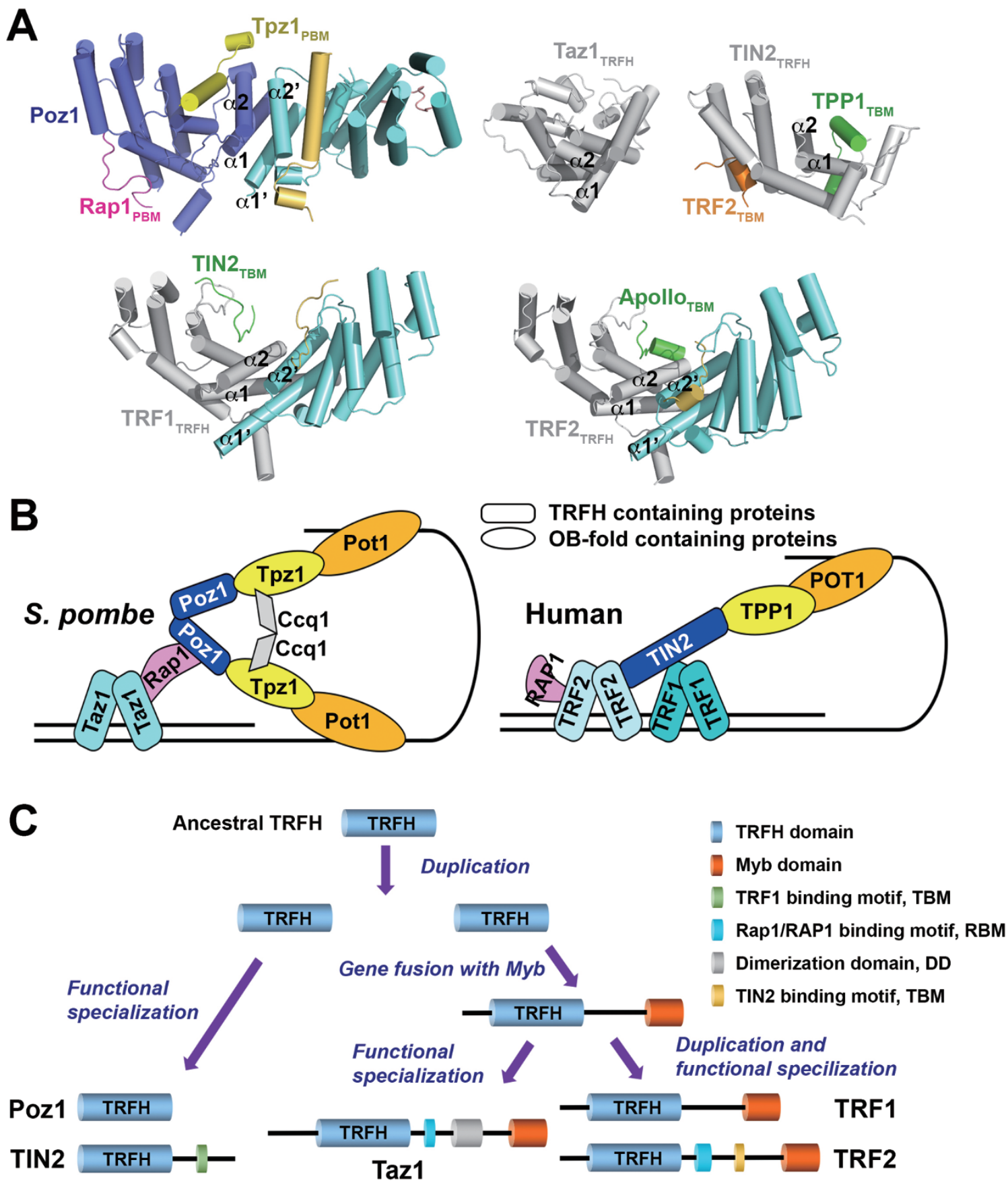

Figure 6 Structural conservation of Poz1 with other shelterin proteins. (A) Ribbon diagrams of Poz1-Tpz1 $1_{\mathrm{PBM}}-\mathrm{Rap}_{\mathrm{PBM}}$, Taz-

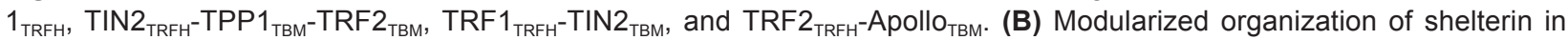
both fission yeast and humans. Each of Taz1, Poz1, TRF1, TRF2, and TIN2 contains a TRFH domain, which is a dimer in Poz1, TRF1, and TRF2 and monomer in Taz1 and TIN2. The dimer of full-length Taz1 is mediated by its dimerization domain (DD). Binding to telomeric dsDNA is exerted by Myb domains in Taz1, TRF1, and TRF2. In the ssDNA region, the OB-fold is the predominant module in Tpz1-Pot1 and TPP1-POT1. (C) Evolutionary model of TRFH-containing shelterin proteins. In the proposed model, an ancestral TRFH gene was first duplicated and the resulting paralogs were both retained in the descendants. In one evolutionary branch, the TRFH gene underwent functional specialization as a bridging molecule (Poz1 in S. pombe and TIN2 in humans) to connect the dsDNA and ssDNA regions of telomeres. In the other evolutionary branch, TRFH became fused with a Myb gene and underwent functional specialization as a dimer to mediate binding with dsDNA regions of telomeres and to function as a protein-protein interacting module to recruit different factors to telomeres (Taz1 in $S$. pombe and TRF1 and TRF2 in humans after another gene duplication). 
tary information, Figure S13), suggesting that Poz1 and TIN2N might have similar structures. Indeed, the crystal structure of TIN2N complexed with TIN2-binding motifs of TPP1 and TRF2 confirmed that TIN2N indeed adopts an $\alpha$-helical architecture that highly resembles the structures of Poz1 and the TRFH domains of TRF1, TRF2, and Taz1 (Figure 6A; Supplementary information, Figure S11) [66], suggesting that similar to Poz1, TIN2 also contains a TRFH domain (TIN2 $2_{\mathrm{TRFH}}$, residues 1-200). Of note, unlike Poz1 and TRFH domains of TRF1 and TRF2, TIN $2_{\text {TRFH }}$ adopts a monomeric conformation (Figure $6 \mathrm{~A}$ ).

\section{Structural features of TRFH domains}

Of all five available TRFH domain structures, four (TRF1, TRF2, TIN2, and Poz1) were crystalized with their respective binding partners $[36,66]$ (Figure 6A). Besides the $\alpha$-helical architecture, the most notable common feature of all these structures is that these TRFH domains employ the same concave groove formed by equivalent helices to bind a short motif of their interacting proteins (Supplementary information, Figure S12). This observation strongly suggests that the TRFH domain is an evolutionarily conserved protein-protein interaction module at telomeres. Given that Taz1 also mediates interactions with multiple proteins [20, 52, 53], it is possible that Tazl could also utilize the same site to interact with some of its binding partners.

The original definition of the TRFH domain was based on the structures of TRF $1_{\mathrm{TRFH}}$ and TRF $2_{\mathrm{TRFH}}$ [49], and homodimerization was considered as a common domain feature. However, the monomeric structures of the TRFH domains of Taz1 and TIN2 are clearly opposed to this notion [48, 66] (Figure 6A). Therefore, homodimerization of TRFH domain might be a feature gained later during evolution or it could be a feature of the ancestral TRFH domain that was in some cases lost later. Further structures of TRFH domains from other species may provide clues to answer this question.

An interesting feature of TRF2 is that it wraps DNA around its TRFH domain through a set of lysine residues to control the DNA topology in human cells [54]. DNA-wrapping lysine residues of TRF $2_{\mathrm{TRFH}}$ are either conserved, replaced by an arginine, or slightly shifted in the structure of TRF $1_{\mathrm{TRFH}}$, consistent with the fact that TRF $1_{\text {TRFH }}$ is also capable of condensing DNA [54]. But this DNA-condensing activity is inhibited by the N-terminal acidic domain of TRF1 [54]. Inspection of the surface residues of the TRFH domains of Poz1, Taz1, and TIN2 did not reveal basic residues at the equivalent positions, suggesting that they probably are not capable of condensing DNAs and that the DNA-wrapping activity of TRF $2_{\text {TRFH }}$ is not a common feature of TRFH domains.

\section{Evolution of S. pome and human shelterin proteins}

The discovery of structural resemblance between Poz1 and the TRFH domains of TRF1, TRF2, Taz1, and TIN2 suggests a plausible model for the evolution of shelterin proteins in fission yeast and humans. In this model, an ancestral single TRFH-domain gene is presumed to undergo gene duplication followed by fixation to yield two paralogs (Figure 6C). One paralog protein has evolved to become the $S$. pombe Poz1 and human TIN2 proteins that, respectively, interact with telomeric single-stranded region proteins, Tpz1 and TPP1 (Figure 6C). By contrast, the other paralog has undergone gene fusion with an ancestral Myb domain-containing gene to yield a hybrid gene that is responsible for the specific recognition of double-stranded telomeric DNAs and other protein factors important for telomere protection and regulation (Figure 6C). In humans, this fusion protein then underwent another gene duplication, and sub-functionalization of the two paralogs resulted in a pair of telomere-capping proteins, TRF1 and TRF2, that share the same architecture but with non-redundant activities (Figure 6C). Although it is possible to imagine alternative evolutionary pathways, our model is relatively parsimonious and invokes just two major events to account for the disparate collection of present day TRFH-containing telomere proteins in fission yeast and humans. In organisms that have a shelterin complex, the Poz1/TIN2 and Taz1/TRF1/ TRF2 orthologs should also harbor TRFH-like domains. Future structural studies of these proteins will be necessary to test the validity of this and related hypotheses.

\section{Materials and Methods}

\section{Protein expression and purification}

S. pombe Poz1 (residues 2-249 or 2-249 with 71-83 deletion) and Tpz1 $1_{\mathrm{PBM}}$ (residues 478-508) were cloned into pMAL-C2X vector with an MBP protein fused at the N-terminus and a modified $\mathrm{pET} 28 \mathrm{a}$ vector with a SUMO protein fused at the N-terminus after the $6 \times$ His tag, respectively [14]. The Poz1-Tpz $1_{\mathrm{PBM}}$ complex was co-expressed in Escherichia coli BL21(DE3). After induction for $18 \mathrm{~h}$ with $0.1 \mathrm{mM} \mathrm{IPTG}$ at $23{ }^{\circ} \mathrm{C}$, the cells were harvested by centrifugation, and the pellets were resuspended in lysis buffer (50 mM Tris- $\mathrm{HCl}, \mathrm{pH} 8.0,300 \mathrm{mM} \mathrm{NaCl}, 10 \%$ glycerol, $1 \mathrm{mM}$ PMSF, $5 \mathrm{mM}$ benzamidine, $1 \mu \mathrm{g} / \mathrm{mL}$ leupeptin, and $1 \mu \mathrm{g} / \mathrm{mL}$ pepstatin). The cells were then lysed by sonication, and the debris was removed by ultracentrifugation. The supernatant was mixed with Ni-NTA agarose beads (Qiagen) and rocked for $2 \mathrm{~h}$ at $4{ }^{\circ} \mathrm{C}$ before elution with $250 \mathrm{mM}$ imidazole. Then, ULP1 and PreScission proteases were added to remove the His-SUMO and MBP tags in an incubation at $4{ }^{\circ} \mathrm{C}$ overnight. The proteins were further purified by Mono-Q and then rebound with Amylose resin (New England Biolabs) to remove the trace MBP tag. After a final step of gel-fil- 
tration chromatography on a HiLoad Superdex200 column equilibrated with $25 \mathrm{mM}$ Tris- $\mathrm{HCl}, \mathrm{pH} 8.0,150 \mathrm{mM} \mathrm{NaCl}$, and $2 \mathrm{mM}$ dithiothreitol, the purified proteins were concentrated to $25 \mathrm{mg} / \mathrm{mL}$ and stored at $-80{ }^{\circ} \mathrm{C}$. The samples of fusion protein Poz1-Rap $1_{\mathrm{PBM}}$ in complex with Tpz1 $1_{\mathrm{PBM}}$ and mutant Poz1-Tpz $1_{\mathrm{PBM}}$ complexes were purified similarly to those described above.

S. pombe Rap $1_{\text {PBM }}$ (residues 466-491) was cloned into pGEX-6P1 vector with a GST protein fused at the N-terminus and expressed in $E$. coli BL21(DE3). After induction for $18 \mathrm{~h}$ with $0.1 \mathrm{mM}$ IPTG at $23{ }^{\circ} \mathrm{C}$, the cells were harvested by centrifugation, and pellets were resuspended in lysis buffer as described above. The cells were then lysed by sonication, and the debris was removed by ultracentrifugation. The supernatant was mixed with glutathione Sepharose-4B beads (GE Healthcare) and rocked overnight at $4{ }^{\circ} \mathrm{C}$ before elution with $15 \mathrm{mM}$ reduced glutathione (Sigma). The PreScission protease was then added to remove the GST tag by incubation at $4{ }^{\circ} \mathrm{C}$ overnight. Rap $1_{\mathrm{PBM}}$ was further purified by gel-filtration chromatography equilibrated with $100 \mathrm{mM}$ ammonium bicarbonate. The purified proteins were concentrated using a Speed Vac system and then lyophilized. The lyophilization products were then resuspended in water at a concentration of $25 \mathrm{mg} / \mathrm{mL}$ and stored at $-80^{\circ} \mathrm{C}$.

$S$. pombe full-length Pot1 and Tpz1 were cloned into modified Bac-to-Bac vectors containing an N-terminal $6 \times$ His tag and an N-terminal GST tag, respectively. Full-length Ccq1 and Poz1 fused with Rap $1_{\text {PBM }}$ were cloned into modified Bac-to-Bac vectors containing no tag. For Ccq1-Tpz1-Pot1-Poz1_Rap1 $1_{\mathrm{PBM}}$ complex expression, High Five insect cells were infected at $\sim 3 \times 10^{6}$ cells $/ \mathrm{mL}$ with a multiplicity of infection of 10 plaque-forming unit $/ \mathrm{mL}$ recombinant baculovirus. The cells were harvested after $72 \mathrm{~h}$ by centrifugation. The pellets were resuspended in lysis buffer $(50 \mathrm{mM}$ Tris- $\mathrm{HCl}, \mathrm{pH}$ 8.0, $150 \mathrm{mM} \mathrm{NaCl}, 10 \%$ glycerol, $1 \mathrm{mM}$ PMSF, $5 \mathrm{mM}$ benzamidine, $1 \mu \mathrm{g} / \mathrm{mL}$ leupeptin, and $1 \mu \mathrm{g} / \mathrm{mL}$ pepstatin). The cells were then lysed by sonication, and the debris was removed by ultracentrifugation. The supernatant was mixed with Ni-NTA agarose beads (Qiagen) and rocked for $2 \mathrm{~h}$ at $4{ }^{\circ} \mathrm{C}$ before elution with $250 \mathrm{mM}$ imidazole. The protein sample was then purified with glutathione Sepharose-4B beads (GE Healthcare) and rocked overnight at 4 ${ }^{\circ} \mathrm{C}$ before elution with $15 \mathrm{mM}$ reduced glutathione (Sigma). PreScission protease was then added to remove the N-terminal $6 \times$ His and GST tags. The proteins were further purified by Mono-Q and gel-filtration chromatography equilibrated with $25 \mathrm{mM}$ Tris- $\mathrm{HCl}$ $\mathrm{pH}$ 8.0, $150 \mathrm{mM} \mathrm{NaCl}$, and $5 \mathrm{mM}$ dithiothreitol. Mutant complex was purified similarly.

Crystallization, data collection, and structure determination Crystals of SeMet-labeled Poz1-Tpz $1_{\mathrm{PBM}}$ complex were grown by sitting-drop vapor diffusion at $4{ }^{\circ} \mathrm{C}$. The precipitant well solution consisted of $0.1 \mathrm{M}$ Bis-Tris, pH 5.5, 2.0 M Ammonium sulfate. Crystals were gradually transferred into a harvesting solution containing 0.1 M Bis-Tris, pH 5.5, 2.2 M Ammonium sulfate, 25\% glycerol, followed by flash-freezing in liquid nitrogen for storage. Data sets were collected under cryogenic conditions $(100 \mathrm{~K})$ at the Shanghai Synchrotron Radiation Facility (SSRF) beamlines BL18U1 and BL19U1. A 2.5- $\AA$ data set of the Poz1-Tpz $1_{\mathrm{PBM}}$ complex was collected at the Se peak wavelength $(0.97860 \AA)$ and processed by HKL3000 [55]. Seven selenium atoms were located and refined, and the single-wavelength anomalous diffraction data phases were calculated using Phenix [56]. The initial SAD map was substantially improved by solvent flattening. The model was then refined using Refmac5 [57], together with manual building in Coot [58]. In the final Ramachandran plot, the favored and allowed residues are $97.6 \%$ and $100.0 \%$, respectively. All the crystal structural figures were generated using PyMOL [59].

Crystals of the Rap $1_{\mathrm{PBM}}-\mathrm{Poz} 1-\mathrm{Tpz} 1_{\mathrm{PBM}}$ complex were grown by sitting-drop vapor diffusion at $4{ }^{\circ} \mathrm{C}$. The precipitant well solution consisted of $0.2 \mathrm{M}$ potassium nitrate, 20\% (w/v) PEG3350. Crystals were gradually transferred into a harvesting solution containing $0.2 \mathrm{M}$ potassium nitrate, 25\% (w/v) PEG3350, 25\% glycerol, followed by flash-freezing in liquid nitrogen for storage. Data sets were collected under cryogenic conditions $(100 \mathrm{~K})$ at the Shanghai Synchrotron Radiation Facility (SSRF) beamlines BL18U1 and BL19U1 and processed by HKL3000 [55]. The Rap $1_{\mathrm{PBM}^{-}}$-Poz1-Tpz $1_{\mathrm{PBM}}$ ternary complex structure was solved by molecular replacement using the Poz1-Tpz $1_{\mathrm{PBM}}$ binary complex structure as the searching model. The model was then refined using Refmac5 [57], together with manual building in Coot [58]. In the final Ramachandran plot, the favored and allowed residues are $96.4 \%$ and $100.0 \%$, respectively. All the crystal structural figures were generated using PyMOL [59].

\section{Isothermal titration calorimetry}

The equilibrium dissociation constant of the interaction between Poz1-Tpz $1_{\mathrm{PBM}}$ and Rap1 $1_{\mathrm{PBM}}$ was determined using a MicroCal iTC200 Calorimeter (Malvern). The binding enthalpies were measured at $20^{\circ} \mathrm{C}$ in $25 \mathrm{mM}$ Tris- $\mathrm{HCl}, \mathrm{pH} 8.0$, and $150 \mathrm{mM} \mathrm{NaCl}$. Two independent experiments were performed for every interaction described here. ITC data were subsequently analyzed and fitted using Origin 7 software (OriginLab).

\section{Yeast two-hybrid assay}

The yeast two-hybrid assays were performed as described previously [60]. Briefly, the L40 strain was transformed with pBTM116 and pACT2 (Clonetech) fusion plasmids, and colonies harboring both plasmids were selected on -Leu -Trp plates. The $\beta$-galactosidase activities were measured by a liquid assay.

\section{Strains and general techniques for fission yeast}

The growth media, basic genetics, and biochemical techniques have been previously described [61, 62]. The yeast strains used in this study are summarized in Supplementary information, Table S1. For the deletions of the $\mathrm{rapl}^{+}$and $\mathrm{pozl}^{+}$genes, each open reading frame was replaced with the marker genes by homologous recombination [63]. Mutations in the $\mathrm{rapl}^{+}$or $\mathrm{pozl}^{+}$genes were created by PCR, and each mutated DNA fragment was used for transformation of the rapl::ura $4^{+}$or poz $1:: u r a 4^{+}$strains to replace the $u r a 4^{+}$cassette with mutated DNA. C-terminal tagging of $p o z 1^{+}$or $t p z 1^{+}$gene was carried out by the insertion of a tag with kanMX6 $\left(\mathrm{kan}^{\prime}\right)$ or hygMX6 $\left(\mathrm{hyg}^{\prime}\right)$ cassettes at chromosomal pozl $1^{+}$or $t p z 1^{+}$gene loci [63].

\section{Telomere Southern blot analysis}

Genomic DNA was digested with ApaI and transferred to Hybond $\mathrm{N}^{+}$nylon membranes (GE Healthcare), and telomere repeats were probed by the ApaI-EcoRI fragment of pAMP1 [64], which contains the $300 \mathrm{bp}$ of telomeric sequence.

\section{Reverse transcription-quantitative PCR}

For detection of the mRNA levels in each strain, reverse transcription and quantitative real-time PCR were performed as de- 
scribed previously [25].

\section{Pulse field gel electrophoresis}

PFGE for detection of telomere end fusion was carried out as described [29]. Cells were grown in YES (Figure 5E), or in EMM with nitrogen and then transferred to EMM without nitrogen for $24 \mathrm{~h}$ to arrest cells in G1 phase (Figure 5C). Genomic DNAs were digested by NotI and probed by a telomere probe (Figure $5 \mathrm{C}$ ) or a probe mixture, which recognizes L, I, M, and C fragments (Figure $5 \mathrm{E})$.

\section{Immunoblotting}

Anti-Flag (F3165; Sigma) and anti-PSTAIR (P7962; Sigma) antibodies were used to detect Poz1-Flag and Cdc2, respectively. Antibody to the C-terminal region of Rap1 obtained as described previously was used to detect Rap1 [65].

\section{Data availability}

Coordinates and structure factors have been deposited in the Protein Data Bank under accession codes 5XXE (Poz1-Tpz $\left.1_{\mathrm{PBM}}\right)$ and $5 \mathrm{XXF}\left(\operatorname{Rap}_{\mathrm{PBM}}-\mathrm{Poz} 1-\mathrm{Tpz} 1_{\mathrm{PBM}}\right)$.

\section{Acknowledgments}

We thank L Wu, D Yao, and R Zhang from BL18U1 and BL19U1 beamlines at NCPSS and Shanghai Synchrotron Radiation Facility (SSRF) for help with crystal data collection and processing, and Y Takeshita for technical assistance. This work was supported by grants from the Ministry of Science and Technology of China (2013CB910402 to ML), the National Natural Science Foundation of China (31330040 and 31525007 to ML, 31500625 to JW, and 31470737 and 31670748 to YC), the Strategic Priority Research Program of the Chinese Academy of Sciences (XDB08010201) to ML and YC, the Basic Research Project of Shanghai Science and Technology Commission (14JC1407200) to $\mathrm{YC}$, the Youth Innovation Promotion Association of the Chinese Academy of Sciences to JW, and Japan Society for the Promotion of Science (JSPS) KAKENHI (17H03606) to JK. This work was performed in part under the International Cooperative Research Program of Institute of Protein Research, Osaka University, ICRa17-04.

\section{Author Contributions}

$\mathrm{ML}, \mathrm{JK}$, and YC conceived this study. JX and HC carried out the bulk of the experiments; HS purified the Ccq1-Tpz1-Pot1Poz1_Rap1 $1_{\mathrm{PBM}}$ complex; JX, HC, and JW collected crystallographic data, carried out the crystallographic analysis, and interpreted the results. MT, HI, and JK designed and performed the mutational in vivo analyses. YL purified the mutant Poz1-Tpz $1_{\mathrm{PBM}}$ proteins used for gel-filtration analysis. JX, HC and JW prepared the figures and $\mathrm{ML}, \mathrm{JK}$ and $\mathrm{YC}$ wrote the manuscript.

\section{Competing Financial Interests}

The authors declare no competing financial interests.

\section{References}

1 Cech TR. Beginning to understand the end of the chromo- some. Cell 2004; 116:273-279.

2 Smogorzewska A, de Lange T. Regulation of telomerase by telomeric proteins. Annu Rev Biochem 2004; 73:177-208.

3 Palm W, de Lange T. How shelterin protects mammalian telomeres. Annu Rev Genet 2008; 42:301-334.

4 Blackburn EH. Switching and signaling at the telomere. Cell 2001; 106:661-673.

5 Levis RW. Viable deletions of a telomere from a Drosophila chromosome. Cell 1989; 58:791-801.

6 Artandi SE, Chang S, Lee SL, et al. Telomere dysfunction promotes non-reciprocal translocations and epithelial cancers in mice. Nature 2000; 406:641-645.

7 Armanios M, Blackburn EH. The telomere syndromes. Nat Rev Genet 2012; 13:693-704.

8 de Lange T. Shelterin: the protein complex that shapes and safeguards human telomeres. Genes Dev 2005; 19:2100-2110.

9 Bilaud T, Brun C, Ancelin K, Koering CE, Laroche T, Gilson E. Telomeric localization of TRF2, a novel human telobox protein. Nat Genet 1997; 17:236-239.

10 Broccoli D, Smogorzewska A, Chong L, de Lange T. Human telomeres contain two distinct Myb-related proteins, TRF1 and TRF2. Nat Genet 1997; 17:231-235.

11 Zhong Z, Shiue L, Kaplan S, de Lange T. A mammalian factor that binds telomeric TTAGGG repeats in vitro. Mol Cell Biol 1992; 12:4834-4843.

12 Lei M, Podell ER, Cech TR. Structure of human POT1 bound to telomeric single-stranded DNA provides a model for chromosome end-protection. Nat Struct Mol Biol 2004; 11:12231229.

13 Loayza D, Parsons H, Donigian J, Hoke K, de Lange T. DNA binding features of human POT1: a nonamer 5'-TAGGGTTAG-3' minimal binding site, sequence specificity, and internal binding to multimeric sites. J Biol Chem 2004; 279:13241-13248.

14 Wang F, Podell ER, Zaug AJ, et al. The POT1-TPP1 telomere complex is a telomerase processivity factor. Nature 2007; 445:506-510.

15 Ye JZ, Hockemeyer D, Krutchinsky AN, et al. POT1-interacting protein PIP1: a telomere length regulator that recruits POT1 to the TIN2/TRF1 complex. Genes Dev 2004; 18:16491654.

16 O’Connor MS, Safari A, Xin H, Liu D, Songyang Z. A critical role for TPP1 and TIN2 interaction in high-order telomeric complex assembly. Proc Natl Acad Sci USA 2006; 103:1187411879.

17 Moser BA, Nakamura TM. Protection and replication of telomeres in fission yeast. Biochem Cell Biol 2009; 87:747-758.

18 Miyoshi T, Kanoh J, Saito M, Ishikawa F. Fission yeast Pot1Tpp1 protects telomeres and regulates telomere length. Science 2008; 320:1341-1344.

19 Cooper JP, Nimmo ER, Allshire RC, Cech TR. Regulation of telomere length and function by a Myb-domain protein in fission yeast. Nature 1997; 385:744-747.

20 Kanoh J, Ishikawa F. spRap1 and spRif1, recruited to telomeres by Taz1, are essential for telomere function in fission yeast. Curr Biol 2001; 11:1624-1630.

21 Miller KM, Ferreira MG, Cooper JP. Taz1, Rap1 and Rif1 act both interdependently and independently to maintain telomeres. EMBO J 2005; 24:3128-3135. 
22 Chikashige Y, Hiraoka Y. Telomere binding of the Rap1 protein is required for meiosis in fission yeast. Curr Biol 2001; 11:1618-1623.

23 Baumann P, Cech TR. Pot1, the putative telomere end-binding protein in fission yeast and humans. Science 2001; 292:11711175.

24 Lei M, Podell ER, Baumann P, Cech TR. DNA self-recognition in the structure of Pot1 bound to telomeric single-stranded DNA. Nature 2003; 426:198-203.

25 Fujita I, Tanaka M, Kanoh J. Identification of the functional domains of the telomere protein Rap1 in Schizosaccharomyces pombe. PLoS One 2012; 7:e49151.

26 Harland JL, Chang YT, Moser BA, Nakamura TM. Tpz1Ccq1 and Tpz1-Poz1 interactions within fission yeast shelterin modulate Ccq1 Thr93 phosphorylation and telomerase recruitment. PLoS Genet 2014; 10:e1004708.

27 Moser BA, Chang YT, Kosti J, Nakamura TM. Tel1 ATM and Rad3ATR kinases promote Ccq1-Est1 interaction to maintain telomeres in fission yeast. Nat Struct Mol Biol 2011; 18:14081413.

28 Tomita K, Cooper JP. Fission yeast Ccq1 is telomerase recruiter and local checkpoint controller. Genes Dev 2008; 22:3461-3474.

29 Chen Y, Rai R, Zhou ZR, et al. A conserved motif within RAP1 has diversified roles in telomere protection and regulation in different organisms. Nat Struct Mol Biol 2011; 18:213221.

30 Yamazaki H, Tarumoto Y, Ishikawa F. Tel1ATM and Rad3ATR phosphorylate the telomere protein Ccq1 to recruit telomerase and elongate telomeres in fission yeast. Genes Dev 2012; 26:241-246.

31 Sugiyama T, Cam HP, Sugiyama R, et al. SHREC, an effector complex for heterochromatic transcriptional silencing. Cell 2007; 128:491-504.

32 Wang J, Cohen AL, Letian A, et al. The proper connection between shelterin components is required for telomeric heterochromatin assembly. Genes Dev 2016; 30:827-839.

33 Konig P, Giraldo R, Chapman L, Rhodes D. The crystal structure of the DNA-binding domain of yeast RAP1 in complex with telomeric DNA. Cell 1996; 85:125-136.

34 Court R, Chapman L, Fairall L, Rhodes D. How the human telomeric proteins TRF1 and TRF2 recognize telomeric DNA: a view from high-resolution crystal structures. EMBO Rep 2005; 6:39-45.

35 Chen $\mathrm{C}, \mathrm{Gu}$ P, Wu J, et al. Structural insights into POT1TPP1 interaction and POT1 C-terminal mutations in human cancer. Nat Commun 2017; 8:14929.

36 Chen Y, Yang Y, van Overbeek M, et al. A shared docking motif in TRF1 and TRF2 used for differential recruitment of telomeric proteins. Science 2008; 319:1092-1096.

37 Jun HI, Liu J, Jeong H, Kim JK, Qiao F. Tpz1 controls a telomerase-nonextendible telomeric state and coordinates switching to an extendible state via Ccq1. Genes Dev 2013; 27:1917-1931.

38 Chang YT, Moser BA, Nakamura TM. Fission yeast shelterin regulates DNA polymerases and $\operatorname{Rad} 3$ (ATR) kinase to limit telomere extension. PLoS Genet 2013; 9:e1003936.

39 Pan L, Hildebrand K, Stutz C, Thoma N, Baumann P. Minishelterins separate telomere length regulation and end protec- tion in fission yeast. Genes Dev 2015; 29:1164-1174.

40 Nimmo ER, Pidoux AL, Perry PE, Allshire RC. Defective meiosis in telomere-silencing mutants of Schizosaccharomyces pombe. Nature 1998; 392:825-828.

41 Tadeo X, Wang J, Kallgren SP, et al. Elimination of shelterin components bypasses RNAi for pericentric heterochromatin assembly. Genes Dev 2013; 27:2489-2499.

42 Hansen KR, Ibarra PT, Thon G. Evolutionary-conserved telomere-linked helicase genes of fission yeast are repressed by silencing factors, RNAi components and the telomere-binding protein Taz1. Nucleic Acids Res 2006; 34:78-88.

43 Ferreira MG, Cooper JP. Two modes of DNA double-strand break repair are reciprocally regulated through the fission yeast cell cycle. Genes Dev 2004; 18:2249-2254.

44 Carneiro T, Khair L, Reis CC, et al. Telomeres avoid end detection by severing the checkpoint signal transduction pathway. Nature 2010; 467:228-232.

45 Flory MR, Carson AR, Muller EG, Aebersold R. An SMC-domain protein in fission yeast links telomeres to the meiotic centrosome. Mol Cell 2004; 16:619-630.

46 Moser BA, Subramanian L, Khair L, Chang YT, Nakamura TM. Fission yeast Tel1ATM and Rad3ATR promote telomere protection and telomerase recruitment. PLoS Genet 2009; 5:e1000622.

47 Scott H, Kim JK, Yu C, Huang L, Qiao F, Taylor DJ. Spatial organization and molecular interactions of the Schizosaccharomyces pombe Ccq1-Tpz1-Poz1 shelterin complex. J Mol Biol 2017; 429:2863-2872.

48 Deng W, Wu J, Wang F, et al. Fission yeast telomere-binding protein Taz1 is a functional but not a structural counterpart of human TRF1 and TRF2. Cell Res 2015; 25:881-884.

49 Fairall L, Chapman L, Moss H, de Lange T, Rhodes D. Structure of the TRFH dimerization domain of the human telomeric proteins TRF1 and TRF2. Mol Cell 2001; 8:351-361.

50 Cooper JP, Watanabe Y, Nurse P. Fission yeast Taz1 protein is required for meiotic telomere clustering and recombination. Nature 1998; 392:828-831.

51 Xin H, Liu D, Wan M, et al. TPP1 is a homologue of ciliate TEBP-beta and interacts with POT1 to recruit telomerase. $\mathrm{Na}$ ture 2007; 445:559-562.

52 Ueno M, Kurokawa R, Renauld H, et al. Schizosaccharomyces pombe taf1+ is required for nitrogen starvation-induced sexual development and for entering the dormant GO state. Curr Genet 2001; 38:307-313.

53 Spink K, Ho JC, Tanaka K, Watts FZ, Chambers A. The telomere-binding protein Tazlp as a target for modification by a SUMO-1 homologue in fission yeast. Biochem Genet 2005; 43:103-117.

54 Benarroch-Popivker D, Pisano S, Mendez-Bermudez A, et al. TRF2-mediated control of telomere DNA topology as a mechanism for chromosome-end protection. Mol Cell 2016; 61:274-286.

55 Minor W, Cymborowski M, Otwinowski Z, Chruszcz M. HKL-3000: the integration of data reduction and structure solution--from diffraction images to an initial model in minutes. Acta Crystallogr D Biol Crystallogr 2006; 62:859-866.

56 Adams PD, Afonine PV, Bunkoczi G, et al. PHENIX: a comprehensive Python-based system for macromolecular structure solution. Acta Crystallogr D Biol Crystallogr 2010; 66:213- 
221.

57 Murshudov GN, Vagin AA, Dodson EJ. Refinement of macromolecular structures by the maximum-likelihood method. Acta Crystallogr D Biol Crystallogr 1997; 53:240-255.

58 Emsley P, Lohkamp B, Scott WG, Cowtan K. Features and development of Coot. Acta Crystallogr D Biol Crystallogr 2010; 66:486-501.

59 Schrodinger L. The PyMOL Molecular Graphics System, Version 1.8. 2015.

60 Sun J, Yang Y, Wan K, et al. Structural bases of dimerization of yeast telomere protein $\mathrm{Cdc13}$ and its interaction with the catalytic subunit of DNA polymerase alpha. Cell Res 2011; 21:258-274.

61 Moreno S, Klar A, Nurse P. Molecular genetic analysis of fission yeast Schizosaccharomyces pombe. Methods Enzymol 1991; 194:795-823.

62 Forsburg SL, Rhind N. Basic methods for fission yeast. Yeast 2006; 23:173-183.
63 Bahler J, Wu JQ, Longtine MS, et al. Heterologous modules for efficient and versatile PCR-based gene targeting in Schizosaccharomyces pombe. Yeast 1998; 14:943-951.

64 Matsuura A, Naito T, Ishikawa F. Genetic control of telomere integrity in Schizosaccharomyces pombe: $\operatorname{rad} 3(+)$ and tel1(+) are parts of two regulatory networks independent of the downstream protein kinases chk1(+) and cds1(+). Genetics 1999; 152:1501-1512.

65 Fujita I, Nishihara Y, Tanaka M, et al. Telomere-nuclear envelope dissociation promoted by Rap1 phosphorylation ensures faithful chromosome segregation. Curr Biol 2012; 22:19321937.

$66 \mathrm{Hu} \mathrm{C}$, Rai R, Huang C, et al. Structural and functional analyses of the mammalian TIN2-TPP1-TRF2 telomeric complex. Cell Res 2017; 27:1485-1502.

(Supplementary information is linked to the online version of the paper on the Cell Research website.) 